\title{
Flow properties for Young-measure solutions of semilinear hyperbolic problems
}

\author{
Alexander Mielke \\ Institut für Angewandte Mathematik, \\ Universität Hannover, Welfengarten 1, D-30167 Hannover \\ (mielke@ifam.uni-hannover.de)
}

(MS received 14 May 1997; accepted 15 July 1998)

\begin{abstract}
For hyperbolic systems in one spatial dimension $\partial_{t} u+C \partial_{x} u=f(u), u(t, x) \in \mathbb{R}^{d}$, we study sequences of oscillating solutions by their Young-measure limit, $\mu$, and develop tools to study the evolution of $\mu$ directly from the Young measure, $\nu$, of the initial data. For $d \leqslant 2$ we construct a flow mapping, $\mathcal{S}_{t}$, such that $\mu(t)=\mathcal{S}_{t}(\nu)$ is the unique Young-measure solution for initial value $\nu$. For $d \geqslant 3$ we establish existence and uniqueness of Young measures that have product structure, that is the oscillations in direction of the Riemann invariants are independent. Counterexamples show that neither $\mu$ nor the marginal measures of the Riemann invariants are uniquely determined from $\nu$, except if a certain structural interaction condition for $f$ is satisfied. We rely on ideas of transport theory and make use of the Wasserstein distance on the space of probability measures.
\end{abstract}

\section{Introduction}

Whenever partial differential equations allow for highly oscillatory solutions it is desirable to find methods to study the evolution of such oscillations. Since the oscillations can be described by Young measures, it is advantageous to derive evolution equations for them. Such evolution equations can be understood as macroscopic equations for microscopic effects.

Here we consider spatially one-dimensional semilinear hyperbolic systems of first order, given in the form

$$
\partial_{t} u+C \partial_{x} u=f(u),
$$

where $t, x \in \mathbb{R}$ and $u=u(t, x) \in \mathbb{R}^{d}$. The system is called hyperbolic if $C$ is diagonalizable and has only real eigenvalues. Hence, without loss of generality we assume that $C$ is diagonal, namely $C=\operatorname{diag}\left(c_{1}, \ldots, c_{d}\right)$. The whole theory presented below can be generalized from right-hand sides $f(u)$ to $f(t, x, u)$, where $f: \mathbb{R}^{2} \times \mathbb{R}^{d} \rightarrow \mathbb{R}^{d}$ is measurable and globally Lipschitz continuous in $u$. However, for notational simplicity we restrict ourselves to right-hand sides depending solely on $u$.

A solution, $u$, of $(1.1)$ is a function in $L_{\text {loc }}^{\infty}\left(\mathbb{R}^{2}, \mathbb{R}^{d}\right)$ that satisfies (1.1) in the sense of distributions. Our interest lies in sequences $\left(u^{n}\right)_{n \in \mathbb{N}}$ of solutions that have a Young measure (YM) limit $\mu \in \mathrm{YM}_{\mathrm{c}}\left(\mathbb{R}^{2}, \mathbb{R}^{d}\right.$ ), written as $u^{n} \stackrel{\mathrm{YM}}{\longrightarrow} \mu$ (see the beginning of $\S 2$ for a short introduction to $\mathrm{YMs}$ ). 
Definition 1.1. A YM $\mu$ is called a Young-measure solution of (1.1) if it is the YM limit of a sequence $\left(u^{n}\right)_{n \in \mathbb{N}}$ of solutions of $(1.1)$ that is bounded in $L_{\text {loc }}^{\infty}\left(\mathbb{R}^{2}, \mathbb{R}^{d}\right)$.

A pair $(\mu, \nu) \in \mathrm{YM}_{\mathrm{c}}\left(\mathbb{R}^{2}, \mathbb{R}^{d}\right) \times \mathrm{YM}_{\mathrm{c}}\left(\mathbb{R}, \mathbb{R}^{d}\right)$ is called a YM solution of the Cauchy problem for (1.1) if there exists a sequence $\left(u^{n}\right)_{n \in \mathbb{N}}$ of solutions of (1.1) that is bounded in $L_{\mathrm{loc}}^{\infty}\left(\mathbb{R}^{2}, \mathbb{R}^{d}\right)$ and satisfies $u^{n} \stackrel{\mathrm{YM}}{\longrightarrow} \mu$ in $\mathrm{YM}_{\mathrm{C}}\left(\mathbb{R}^{2}, \mathbb{R}^{d}\right)$ and $u^{n}(0, \cdot) \stackrel{\mathrm{YM}}{\longrightarrow} \nu$ in $\mathrm{YM}_{\mathrm{c}}\left(\mathbb{R}, \mathbb{R}^{d}\right)$.

This definition implies that the set of all YM solutions of (1.1) is closed with respect to the weak* topology. There is also the notion of 'measure-valued solution' due to DiPerna ( [5]) which is the same as ours if it is used in the strong sense, as for instance in [6]. However, often the notion of measured-valued solutions is used in a much weaker sense, enforcing equations only for simple averages, see remark 3.2 and the definition on p. 387 of [4]. To avoid misunderstandings we thus stay with our naming.

The Cauchy problem in the sense of YM solutions is to find $\mu$ for a given $\nu$. One such $\mu$ is easily constructed as follows. Choose a sequence $v^{n} \in L_{\text {loc }}^{\infty}\left(\mathbb{R}, \mathbb{R}^{d}\right)$ with $v^{n} \stackrel{\mathrm{YM}}{\longrightarrow} \nu$, then there exist weak solutions $u^{n} \in L_{\text {loc }}^{\infty}\left(\mathbb{R}^{2}, \mathbb{R}^{d}\right)$ of $(1.1)$ with $u^{n}(0, x)=v^{n}(x)$. The fundamental theorem for YMs guarantees the existence of $\mu$ such that $u^{n} \stackrel{Y M}{\longrightarrow} \mu$, after extracting a subsequence. The following questions are in order.

(Q1) Is $\mu$ uniquely determined by $\nu$ ?

(Q2) How is $\mu(0, \cdot)$ related to $\nu$ ?

(Q3) Is there an evolution equation for $\mu$ such that it can be calculated directly from $\nu$, without using sequences $\left(u^{n}\right)_{n \in \mathbb{N}}$ ?

Such evolution equations for $\mu$ allow us to describe microscopic effects macroscopically, and they are important if the qualitative behaviour of $\mu(t)$ for $t \rightarrow \infty$ is to be studied, see $[21,25,26]$.

Similar questions were already treated in [18-23] and $[7,13]$; however, there YM solutions are described by studying the evolution of their moments

$$
E(t, x, \alpha)=\left\langle\mu(t, x, \mathrm{~d} u), \prod_{j=1}^{d}\left(u_{j}\right)^{\alpha_{j}}\right\rangle_{\mathbb{R}^{d}},
$$

$\alpha \in \mathbb{N}_{0}^{d}$. If all moments $E(t, x, \alpha), \alpha \in \mathbb{N}_{0}^{d}$, are known, then the measure $\mu(t, x)$ can be uniquely reconstructed. Yet, useful evolution equations for the moments can only be obtained for certain polynomial right-hand sides. Our analysis is much closer to the work in $[10,11]$, where equations from nonlinear optics are studied.

To explain our main ideas and results we need the notion of marginal measures. If $u^{n} \stackrel{\mathrm{YM}}{\longrightarrow} \mu \in \mathrm{YM}_{\mathrm{c}}\left(\mathbb{R}^{2}, \mathbb{R}^{d}\right)$, then the marginal measures $\mu_{j} \in \mathrm{YM}_{\mathrm{c}}\left(\mathbb{R}^{2}, \mathbb{R}\right)$ are either defined by $u_{j}^{n} \stackrel{\mathrm{YM}}{\longrightarrow} \mu_{j}$ or by $\left\langle\mu_{j}\left(\mathrm{~d} v_{j}\right), \psi_{j}\left(v_{j}\right)\right\rangle_{v_{j} \in \mathbb{R}}=\left\langle\mu(\mathrm{d} v), \psi_{j}\left(v_{j}\right)\right\rangle_{v \in \mathbb{R}^{d}}$. From the vector of marginals, $\bar{\mu}=\left(\mu_{1}, \ldots, \mu_{d}\right) \in\left(\mathrm{YM}_{\mathrm{c}}\left(\mathbb{R}^{2}, \mathbb{R}\right)\right)^{d}$, it is generally not possible to reconstruct the full measure $\mu$, unless it is equal to the product measure $\otimes_{j=1}^{d} \mu_{j}$. Notice that our choice $C=\operatorname{diag}\left(c_{1}, \ldots, c_{d}\right)$ implies that $\mu_{j}$ is the marginal measure associated to the Riemann invariant $u_{j}$ of $\partial_{t} u+C \partial_{x} u=f(u)$. 
A partial answer to the questions Q1-Q3 is given in $\S 8$ where we show that, for Lipschitz continuous $f$ and initial conditions $\mu_{j}(0, \cdot)=\nu_{j}$, system (1.1) always has a unique product-measure solution, that is $\mu(t, x)=\otimes_{j=1}^{d} \mu_{j}(t, x)$. Moreover, the vector of marginals $\bar{\mu}$ solves the evolution equation

$$
\partial_{t} \mu_{j}+c_{j} \partial_{x} \mu_{j}+\partial_{u_{j}} \mu_{j}\left[f_{j}^{(\bar{\mu})}\left(t, x, u_{j}\right)\right]=0, \quad \mu_{j}(0, x)=\nu_{j}(x), \quad \text { for } j=1, \ldots, d,
$$

where $f_{j}^{(\bar{\mu})}$ is obtained from $f_{j}(u)$ by averaging all variables $u_{k}$ except for $u_{j}$, see (3.12). We call this system the non-resonance system associated to (1.1). It will be the organizing centre of this work.

There is a vague analogy between our uniqueness result for product-measure solutions and the uniqueness result in [4] for a parabolic equation for forwardbackward type. There, the 'independence lemma' (4.1) is crucial to deduce uniqueness (see $[4$, remark 1, p. 393]).

Obviously, product-measure solutions generalize the classical solutions $u(t, x)$ in the most natural way as classical solutions are YM solutions in the form $\mu(t, x)=$ $\delta_{u(t, x)}$ and point measures are always product measures. In this sense, (1.2) can be understood as a genuine generalization of (1.1). In light of [11] and of our results one should think of the product-measure structure as the generic case, while other measures occur only in special resonant situations. There is also an entropy argument favouring the set of product-measure solutions. Assume that $\mu$ is either purely discrete or has a density, such that the information entropy is defined. Then the information entropy of $\mu$ is smaller than the information entropy of $\otimes_{1}^{d} \mu_{j}$ with equality, if and only if $\mu$ is the product measure (see [1, theorem 1.4.3]). In our example 6.2, we make a small comparison of the information entropy for a general YM solution to the product-measure solution.

Our existence and uniqueness results for (1.2), given in $\S 4$, avoids the use of the moments $E(t, x, \alpha)$ and is based on ideas from transport theory for Vlasov systems (see, for example, [3]). A similar approach is employed in $[25,26]$ to study the YM solutions of certain regularizations of a non-monotone wave equation. Our solutions are constructed iteratively such that $\bar{\mu}^{n+1}$ is obtained by solving (1.2), where $f_{j}^{(\bar{\mu})}$ is replaced by $g_{j}^{n}=f_{j}^{\left(\bar{\mu}^{n}\right)}$. Thus, the problem for $\bar{\mu}^{n+1}$ is linear and easily solved by transporting $\bar{\nu}$ along the characteristics. The key feature in transport theory is that it is much easier to set up a contraction argument for the mapping $\bar{g}^{n} \mapsto \bar{g}^{n+1}$ in place of the mapping $\bar{\mu}^{n} \mapsto \bar{\mu}^{n+1}$, see the proof of theorem 4.1. Adding up, we can associate with (1.2) a flow group $\left(\mathcal{S}_{t}\right)_{t \in \mathbb{R}}$ on $\left(\mathrm{YM}_{c}(\mathbb{R}, \mathbb{R})\right)^{d}$, such that $\mathcal{S}_{t} \circ \mathcal{S}_{\tau}=\mathcal{S}_{t+\tau}$ and $\bar{\mu}(t)=\mathcal{S}_{t}(\bar{\nu})$ is the unique solution of (1.2). Moreover, $t \mapsto \mathcal{S}_{t}(\nu)$ is weak* continuous.

In dimensions $d \leqslant 2$, the questions Q1-Q3 can be answered completely. For $d=2$ and $c_{1} \neq c_{2}$ and $f$ Lipschitz continuous, the YM $\mu$ is uniquely determined by $\nu$, has the form $\mu=\mu_{1} \otimes \mu_{2}$, and $\left(\mu_{1}, \mu_{2}\right)$ solves (1.2). Moreover, $\mu_{j}(0, \cdot)=\nu_{j}$ implying $\mu(0, \cdot) \neq \nu$ in general. The product structure was already established in $[6,10,21]$, and is a consequence of the div-curl lemma of Murat [12] and Tartar [17]. In [11], the case $d=3$ with wave speeds $c_{j}$ depending on $(t, x)$ is treated: under certain nonresonance conditions (excluding the constant case) it is shown that $\mu=\mu_{1} \otimes \mu_{2} \otimes \mu_{3}$ by using 'trilinear compensated compactness'. 
For $d \geqslant 3$ with constant $c_{j}$, the answer to $\mathrm{Q} 1$ is no, in general. In $\S 6.2$ we present a linear, uncoupled problem $(f(u)=0)$ where the marginals are uniquely determined, but $\mu$ differs from the associated product measure on a bounded open set. This leads us to the following restriction of our view to the marginals.

( $\overline{\mathrm{Q} 1})$ Is $\bar{\mu}=\left(\mu_{1}, \ldots, \mu_{d}\right)$ uniquely determined by $\bar{\nu}=\left(\nu_{1}, \ldots, \nu_{d}\right)$ ?

( $\overline{\mathrm{Q} 2})$ How is $\bar{\mu}(0, \cdot)$ related to $\bar{\nu}$ ?

( $\overline{\mathrm{Q}})$ Is there an evolution equation for $\bar{\mu}$ ?

Theorem 3.5 answers question $\overline{\mathrm{Q} 2}$ with $\bar{\mu}(0, \cdot)=\bar{\nu}$, since the marginals of YM solutions of (1.1) depend weak* continuous on time. Again using the div-curl lemma question $\overline{\mathrm{Q} 1}$ can be answered in the affirmative if the right-hand side $f$ satisfies the interaction condition

$$
f_{j}(u)=\sum_{k=1}^{d} g_{j, k}\left(u_{j}, u_{k}\right), \quad \text { for } j=1, \ldots, d .
$$

Then, the evolution equation for $\bar{\mu}$ is again given by $(1.2)$ and we have $\bar{\mu}(t)=\mathcal{S}_{t}(\bar{\nu})$. This allows us, in particular, to solve all linear problems (see $\S 6.1$ ).

Section 6.3 treats the well-known example (see [10])

$$
\left(\partial_{t}-\partial_{x}\right) u_{1}=0, \quad \partial_{t} u_{2}=2 u_{1} u_{3}, \quad\left(\partial_{t}+\partial_{x}\right) u_{3}=0
$$

which does not satisfy the interaction condition. We explicitly construct two YM solutions $\mu$ and $\eta$ for the same initial data, $\bar{\nu}$, such that $\bar{\mu}$ and $\bar{\eta}$ are different. Hence the answer to question $\overline{\mathrm{Q} 1}$ is no, in general.

In $\S 7$ we establish continuity properties of $\mathcal{S}_{t}$ as a mapping from $\left(\mathrm{YM}_{\mathrm{C}}(\mathbb{R}, \mathbb{R})\right)^{d}$ into itself. In the weak ${ }^{*}$ topology, continuity holds if the interaction condition (1.3) is valid. However, there is another useful topology given by the Wasserstein distance for probability measures:

$$
\mathrm{d}_{\mathrm{W}}(\mu, \eta)=\sup \{|\langle\mu, \psi\rangle-\langle\eta, \psi\rangle|: \operatorname{Lip}(\psi) \leqslant 1\}
$$

see $\S 2$ for more details. This distance is ideally suited for problems involving transport of measures, for instance we obtain

$\operatorname{dist}_{p}\left(\mathcal{S}_{t}(\bar{\nu}), \mathcal{S}_{t}(\bar{\kappa})\right) \leqslant \mathrm{e}^{L|t|} \operatorname{dist}_{p}(\bar{\nu}, \bar{\kappa}), \quad \operatorname{dist}_{p}(\bar{\nu}, \bar{\kappa})=\left(\int_{\mathbb{R}} \mathrm{d}_{\mathrm{W}}(\bar{\nu}(x), \bar{\kappa}(x))^{p} \mathrm{~d} x\right)^{1 / p}$,

where $L$ is the Lipschitz constant of $f$.

Convergence in the Wasserstein metric dist $_{p}$ implies weak ${ }^{*}$ convergence but not vice versa. The interplay between these two topologies plays a crucial role in our existence of product-measure solutions in $\S 8$. Considering initial data $v_{j}^{n}(x)=$ $V_{j}\left(x, n \omega_{j} x\right)$ with $V_{j} \in C(\mathbb{R} \times \mathbb{T}, \mathbb{R})$ we are able to show that

$$
v_{j}^{n} \stackrel{\mathrm{YM}}{\longrightarrow} \gamma_{j} \quad \text { and } \quad u^{n} \stackrel{\mathrm{YM}}{\longrightarrow} \mu=\otimes_{1}^{d} \mu_{j}
$$

whenever $\left(\omega_{1}, \ldots, \omega_{d}\right)$ satisfies a non-resonance condition of small divisor type, cf. lemma 8.2. Here,

$$
\left\langle\gamma_{j}(x), \psi\right\rangle=\int_{\mathbb{T}} \psi\left(V_{j}(x, z)\right) \mathrm{d} z \quad \text { and } \quad\left\langle\mu_{j}(x), \psi\right\rangle=\int_{\mathbb{T}} \psi\left(\tilde{U}_{j}\left(t, x-c_{j} t, z\right)\right) \mathrm{d} z,
$$


where $\tilde{U} \in C\left(\mathbb{R}^{2} \times \mathbb{T}, \mathbb{R}^{d}\right)$ solves the auxiliary system (8.3), which is a direct counterpart to the non-resonance system (1.2). This weak* convergence follows by a direct and lengthy comparison of $u_{j}^{n}(t, x)$ and $\tilde{U}_{j}\left(t, x-c_{j} t, n \omega_{j}\left(x-c_{j} t\right)\right)$; but now we can approximate general initial data $\nu$ by a sequence $\gamma^{m}$ with $\gamma^{m}$ as above, such that $\operatorname{dist}_{1}\left(\gamma^{m}, \nu\right) \rightarrow 0$ for $m \rightarrow \infty$. With (1.5), the associated YM solutions $\mu^{m}=\otimes_{j=1}^{d} \mu_{j}^{m}$ converge to a limit $\mu^{*}$ that is again a product measure. In fact, to show $\mu^{*}=\otimes_{1}^{d} \mu_{j}^{*}$ it is essential to have convergence in the Wasserstein metric, since the set of product measures is not closed in the weak* topology.

\section{Young measures}

We first give the main definitions and facts about Young measures (YM). Throughout this work we are concerned with sequences $u^{n}: \mathbb{R}^{k} \rightarrow \mathbb{R}^{d}$ that are bounded in $L_{\text {loc }}^{\infty}\left(\mathbb{R}^{k}\right)$. That is, for each $R>0$ there exists $C=C(R)$ such that $\left|u^{n}(y)\right| \leqslant C$ for all $n \in \mathbb{N}$ and almost every (a.e.) $y$ with $|y| \leqslant R$ (in the sense of the Lebesgue measure). Thus, we can simplify the notion of YMs for our purposes. We refer to [2] for a general treatment and only give the definitions for our special case.

Let $\mathrm{M}\left(\mathbb{R}^{d}\right)$ be the set of (signed) Radon measures on $\mathbb{R}^{d}$ which is the dual space of $C_{0}^{0}\left(\mathbb{R}^{d}, \mathbb{R}\right)$ (decaying continuous functions) equipped with the supremum norm. The dual pairing is written as

$$
\langle\mu, \psi\rangle=\langle\mu(\mathrm{d} u), \psi(u)\rangle_{u \in \mathbb{R}^{d}}=\int_{u \in \mathbb{R}^{d}} \psi(u) \mu(\mathrm{d} u) .
$$

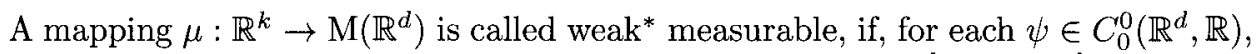
the mapping $x \mapsto\langle\mu(x), \psi\rangle$ is (Lebesgue) measurable. By $\mathrm{P}\left(\mathbb{R}^{d}\right) \subset \mathrm{M}\left(\mathbb{R}^{d}\right)$ we denote the subset of probability measures, i.e. $\mu$ is positive $\left(\langle\mu, \psi\rangle \geqslant 0\right.$ for all $\psi \in C_{0}^{0}\left(\mathbb{R}^{d}, \mathbb{R}\right)$ with $\psi(y) \geqslant 0$ for all $y)$ and has mass $1(\langle\mu, 1\rangle=1)$.

Then, the set of YMs is defined as

$\mathrm{YM}_{\mathrm{c}}\left(\mathbb{R}^{k}, \mathbb{R}^{d}\right)$

$$
=\left\{\mu: \mathbb{R}^{k} \rightarrow \mathrm{P}\left(\mathbb{R}^{d}\right): \mu \text { weak }^{*} \text { measurable, } \operatorname{diam}[\operatorname{sppt}(\mu(\cdot))] \in L_{\mathrm{loc}}^{\infty}\left(\mathbb{R}^{k}\right)\right\} .
$$

The subscript ' $c$ ' is used to denote the property of compact support. As usual $\mu$ is identified with the equivalence class of all mappings $\tilde{\mu}$ that satisfy $\tilde{\mu}(y)=\mu(y)$ almost everywhere. We use the following notion of weak* convergence of YMs:

$$
\begin{aligned}
& \mu^{n} \stackrel{*}{\rightarrow} \mu^{\infty} \Longleftrightarrow \exists R \in L_{\mathrm{loc}}^{\infty}\left(\mathbb{R}^{k}\right): \operatorname{diam}\left[\operatorname{sppt}\left(\mu^{n}(x)\right)\right] \leqslant R(x) \text { for a.e. } x \text { and } \\
& \left(\forall \phi \in C_{\mathrm{c}}^{0}\left(\mathbb{R}^{k}\right), \forall \psi \in C_{0}^{0}\left(\mathbb{R}^{d}\right): \int_{\mathbb{R}^{k}} \phi(y)\left\langle\mu^{n}(y), \psi\right\rangle \mathrm{d} y \longrightarrow \int_{\mathbb{R}^{k}} \phi(y)\left\langle\mu^{\infty}(y), \psi\right\rangle \mathrm{d} y\right) .
\end{aligned}
$$

Here $C_{\mathrm{c}}^{k}\left(\mathbb{R}^{d}, \mathbb{R}\right)$ denotes the functions in $C^{k}\left(\mathbb{R}^{d}, \mathbb{R}\right)$ with compact support. The notion contains an unusual assumption on the support of the sequences that guarantees that also the limit $\mu^{\infty}$ has locally bounded support. For the present purposes this notion of convergence is sufficient, and it should be possible to generalize our theory to YMs without bounded support.

Given a Caratheodory function $\Phi=\Phi(y, u) \in \mathbb{R}^{m}$ (i.e. $\Phi$ is measurable in $y$ for all $u$ and continuous in $u$ for a.e. $y$ ) the pull back of a $\mathrm{YM} \mu \in \mathrm{YM}_{\mathrm{c}}\left(\mathbb{R}^{k}, \mathbb{R}^{m}\right)$ to a $\mathrm{YM} \hat{\mu}=\Phi_{*} \mu \in \mathrm{YM}_{\mathrm{C}}\left(\mathbb{R}^{k}, \mathbb{R}^{d}\right)$ is defined as

$$
\langle\hat{\mu}(y, \mathrm{~d} w), \psi(w)\rangle_{w \in \mathbb{R}^{m}}=\langle\mu(y, \mathrm{~d} u), \psi(\Phi(y, u))\rangle_{u \in \mathbb{R}^{d}}, \quad \text { for all } \psi \in C_{\mathrm{c}}\left(\mathbb{R}^{m}, \mathbb{R}\right) .
$$


A function $u \in L_{\text {loc }}^{\infty}\left(\mathbb{R}^{k}, \mathbb{R}^{d}\right)$ defines a YM $\mu$ via $\mu(y)=\delta_{u(y)}$, where $\delta_{a}$ is the Dirac measure located at $y=a$, i.e. $\left\langle\delta_{a}, \psi\right\rangle=\psi(a)$. For a sequence $\left(u^{n}\right)_{n \in \mathbb{N}}$ we say that $u^{n}$ converges to the YM $\mu$ if $\delta_{u^{n}} \stackrel{*}{\rightarrow} \mu$ and write shortly $u^{n} \stackrel{\mathrm{YM}}{\longrightarrow} \mu$. Every YM $\mu$ can be approximated by $L^{\infty}$ functions, i.e. there exists $u^{n} \in L_{\mathrm{loc}}^{\infty}\left(\mathbb{R}^{k}, \mathbb{R}^{d}\right)$ such that $u^{n} \stackrel{\mathrm{YM}}{\longrightarrow} \mu$. Of course YM limits are only interesting if the sequence $u^{n}$ converges weakly but not strongly, since $u^{n} \rightarrow u^{\infty}$ implies that the associated YM limit is $\mu=\delta_{u} \infty$. The most important consequence of the YM construction is the ability to control the convergence of nonlinear functions of $u^{n}$. For every Caratheodory function $g=g(y, u)$ we have

$$
u^{n} \stackrel{\mathrm{YM}}{\longrightarrow} \mu \Longrightarrow g\left(\cdot, u^{n}(\cdot)\right) \stackrel{\mathrm{YM}}{\longrightarrow} g_{*} \mu .
$$

The fundamental theorem for Young measures (see [2]) states that for every sequence $\left(u^{n}\right)_{n}$ that is bounded in $L_{\mathrm{loc}}^{\infty}\left(\mathbb{R}^{k}, \mathbb{R}^{d}\right)$ there exists a subsequence $\left(u^{n_{m}}\right)_{m}$ and a YM $\mu \in \mathrm{YM}_{\mathrm{c}}\left(\mathbb{R}^{k}, \mathbb{R}^{d}\right)$ such that $u^{n_{m}} \stackrel{\text { YM }}{\longrightarrow} \mu$ for $m \rightarrow \infty$. Here we see an advantage of our more restrictive notion of convergence, since we do not need to worry about mass leaking out to infinity.

A YM $\mu \in \mathrm{YM}_{c}\left(\mathbb{R}^{k}, \mathbb{R}^{d}\right)$ defines a distribution on $\mathbb{R}^{k} \times \mathbb{R}^{d}$ via

$$
C_{\mathrm{c}}^{\infty}\left(\mathbb{R}^{k} \times \mathbb{R}^{d}\right) \ni \Phi \mapsto \int_{\mathbb{R}^{k}}\langle\mu(y, d u), \Phi(y, u)\rangle_{u \in \mathbb{R}^{d}} \mathrm{~d} y \in \mathbb{R} .
$$

Thus, partial derivatives $\partial_{y_{i}} \mu$ and $\partial_{u_{j}} \mu$ are well defined in the sense of distributions. A term $D_{u} \mu(y)[h(y, u)]$ is defined via application of test functions $\Phi(y, u)=$ $\phi(y) \psi(u)$ as

$$
\left.-\int_{y \in \mathbb{R}^{k}} \phi(y)\langle\mu(y, \mathrm{~d} u)), D_{u} \psi(u)[h(y, u)]\right\rangle_{u \in \mathbb{R}^{d}} \mathrm{~d} y .
$$

Here, and further on, it suffices to use test functions in product form $(\Phi(y, u)=$ $\phi(y) \psi(u)$ ) since the set of finite linear combinations of such products is dense in $C_{\mathrm{c}}^{\infty}\left(\mathbb{R}^{k} \times \mathbb{R}^{d}\right)$.

We need the notion of marginal measures and product measures. If

$$
J=\left\{j_{1}, \ldots, j_{m}\right\} \subset\{1, \ldots, d\},
$$

we shortly write $w_{J}$ for $\left(w_{j_{1}}, \ldots, w_{j_{m}}\right) \in \mathbb{R}^{m}$ and the marginal measure $M_{J} \mu$ is defined by

$$
\left\langle\left(M_{J} \mu\right)\left(\mathrm{d} w_{J}\right), \psi\left(w_{J}\right)\right\rangle_{\mathbb{R}^{m}}=\left\langle\mu(\mathrm{d} w), \psi\left(w_{J}\right)\right\rangle_{\mathbb{R}^{d}}, \quad \text { for all } \psi \in C_{\mathrm{c}}^{0}\left(\mathbb{R}^{m}\right) .
$$

If $J=\{j\}$, we shortly write $\mu_{j}$ for $M_{\{j\}} \mu$, which is the YM of the $u_{j}$-component alone, namely $u_{j}^{n} \stackrel{\mathrm{YM}}{\longrightarrow} \mu_{j}$. If $J=\{1, \ldots, d\} \backslash\{j\}$ we shortly write $\mu_{\not \supset j}$. For measures $\mu_{j} \in \mathrm{P}\left(\mathbb{R}^{m_{j}}\right)$ the product measure $\hat{\mu}=\mu_{1} \otimes \cdots \otimes \mu_{n} \in \mathrm{P}\left(\mathbb{R}^{m_{1}+\cdots+m_{n}}\right)$ is defined via

$$
\left\langle\hat{\mu}\left(\mathrm{d}\left(w_{1}, \ldots, w_{n}\right)\right), \psi_{1}\left(w_{1}\right) \cdot \ldots \cdot \psi_{n}\left(w_{n}\right)\right\rangle_{\mathbb{R}^{m}}=\prod_{j=1}^{n}\left\langle\mu_{j}\left(\mathrm{~d} w_{j}\right), \psi_{j}\left(w_{j}\right)\right\rangle_{w_{j} \in \mathbb{R}^{m_{j}}},
$$

for all functions $\psi_{j} \in C_{\mathrm{c}}\left(\mathbb{R}^{m_{j}}, \mathbb{R}\right)$. (The set of linear combinations of functions $\psi_{1}\left(w_{1}\right) \cdot \ldots \cdot \psi_{n}\left(w_{n}\right)$ is dense in $C_{\mathrm{c}}\left(\mathbb{R}^{m}, \mathbb{R}\right)$ defining $\hat{\mu}$ uniquely.) Point measures $\delta_{u(t, x)}$ are simple examples of product measures since

$$
\delta_{u(t, x)}(\mathrm{d} u)=\delta_{u_{1}(t, x)}^{1}\left(\mathrm{~d} u_{1}\right) \otimes \cdots \otimes \delta_{u_{d}(t, x)}^{1}\left(\mathrm{~d} u_{d}\right) .
$$


Throughout this work, it is important to distinguish between the two spaces $X_{1}=\left(\mathrm{YM}_{\mathrm{c}}\left(\mathbb{R}^{k}, \mathbb{R}\right)\right)^{d}$ and $X_{2}=\mathrm{YM}_{\mathrm{c}}\left(\mathbb{R}^{k}, \mathbb{R}^{d}\right)$. The product $\otimes$ is a mapping from $X_{1}$ into $X_{2}$, and taking the one-dimensional marginals gives a mapping

$$
\mathcal{M}:\left\{\begin{aligned}
\mathrm{YM}_{\mathrm{C}}\left(\mathbb{R}^{k}, \mathbb{R}^{d}\right) & \rightarrow\left(\mathrm{YM}_{\mathrm{c}}\left(\mathbb{R}^{k}, \mathbb{R}\right)\right)^{d}, \\
\mu & \mapsto\left(\mu_{1}, \ldots, \mu_{d}\right) .
\end{aligned}\right.
$$

Note that $\mathcal{M} \otimes$ is the identity on $X_{1}$, but $\otimes \mathcal{M}$ projects $X_{2}$ onto the product measures. We use the shorthand notation $\bar{\mu}=\mathcal{M} \mu \in\left(\mathrm{YM}_{\mathrm{c}}\left(\mathbb{R}^{k}, \mathbb{R}\right)\right)^{d}$ for the $d$-tuple of marginals, which is not to be confused with the $d$-dimensional $\mathrm{YM}$ $\mu \in \mathrm{YM}_{c}\left(\mathbb{R}^{k}, \mathbb{R}^{d}\right)$.

An important tool in our transport theory for YM is the following Wasserstein distance $\mathrm{d}_{\mathrm{W}}$ between two probability measures. For measures $\mu_{1}, \mu_{2} \in \mathrm{P}_{\mathrm{c}}\left(\mathbb{R}^{d}\right)$ it is defined via

$$
\mathrm{d}_{\mathrm{W}}\left(\mu_{1}, \mu_{2}\right)=\sup \left\{\left\langle\mu_{1}, \psi\right\rangle-\left\langle\mu_{2}, \psi\right\rangle: \operatorname{Lip}(\psi) \leqslant 1\right\}
$$

According to $[15, \S 5.3]$ this is equivalent to the case $p=1$ for the KantorovichRubinstein functional:

$\operatorname{KR}_{p}\left(\mu_{1}, \mu_{2}\right)=\inf \left\{\left(\left\langle\eta(\mathrm{d} u, \mathrm{~d} v),|u-v|^{p}\right\rangle_{(u, v) \in \mathbb{R}^{2 d}}\right)^{1 / p}: \eta \in \mathrm{P}\left(\mathbb{R}^{2 d}\right), \mathcal{M} \eta=\left(\mu_{1}, \mu_{2}\right)\right\}$,

where $\mathcal{M}$ extracts the two marginal measures with respect to $u$ and $v$, respectively. See also [14,25-27] for other applications of the Wasserstein distances to nonlinear partial differential equations.

From the first definition we see that the Wasserstein distance is easy to handle and works particularly well together with Lipschitz continuous functions, thus allowing us to derive Gronwall estimates for differences of YM solutions. The transport of measures is continuous in the Wasserstein distance but discontinuous in the norm topology, e.g. for $t \mapsto \delta_{t}$ we have $\mathrm{d}_{\mathrm{W}}\left(\delta_{t}, \delta_{s}\right)=|t-s|$ but $\left\|\delta_{t}-\delta_{s}\right\|=2$ for $t \neq s$. Moreover, we have a simple estimate for the Wasserstein distance of product measures.

Lemma 2.1. For $\bar{\mu}, \bar{\eta} \in\left(\mathrm{P}_{\mathrm{c}}(\mathbb{R})\right)^{d}$ we have $\mathrm{d}_{\mathrm{W}}\left(\otimes \mu_{j}, \otimes \eta_{j}\right) \leqslant \sum_{k=1}^{d} \mathrm{~d}_{\mathrm{W}}\left(\mu_{k}, \eta_{k}\right)$.

Proof. Define $\xi^{(k)}=\left[\otimes_{1}^{k} \mu_{j}\right] \otimes\left[\otimes_{k+1}^{d} \eta_{l}\right]$ implying $\xi^{(0)}=\otimes \eta_{l}$ and $\xi^{(k)}=\otimes \mu_{j}$. For $\psi \in C\left(\mathbb{R}^{d}, \mathbb{R}\right)$ let $\psi_{k}=A_{k} \psi \in C(\mathbb{R}, \mathbb{R})$ with

$$
\psi_{k}\left(u_{k}\right)=\left\langle\left[\otimes_{j=1}^{k-1} \mu_{j}\left(\mathrm{~d} u_{j}\right)\right] \otimes\left[\otimes_{k+1}^{d} \eta_{l}\left(\mathrm{~d} u_{l}\right)\right], \psi(u)\right\rangle_{u_{\notin k}} .
$$

Now we estimate

$$
\begin{aligned}
\mathrm{d}_{\mathrm{W}}\left(\otimes \mu_{j}, \otimes \eta_{l}\right) & \leqslant \sum_{k=1}^{d} \mathrm{~d}_{\mathrm{W}}\left(\xi^{(k)}, \xi^{(k-1)}\right) \\
& =\sum_{k=1}^{d} \sup \left\{\left|\left\langle\left[\otimes_{j=1}^{k-1} \mu_{j}\right] \otimes\left(\mu_{k}-\eta_{k}\right) \otimes\left[\otimes_{l=k+1}^{d} \eta_{l}\right], \psi\right\rangle\right|: \operatorname{Lip}(\psi) \leqslant 1\right\} \\
& =\sum_{k=1}^{d} \sup \left\{\left|\left\langle\mu_{k}-\eta_{k}, A_{k} \psi\right\rangle\right|: \operatorname{Lip}(\psi) \leqslant 1\right\} \leqslant \sum_{k=1}^{d} \mathrm{~d}_{\mathrm{W}}\left(\mu_{k}, \eta_{k}\right),
\end{aligned}
$$

where $\operatorname{Lip}\left(A_{k} \psi\right) \leqslant \operatorname{Lip}(\psi)$ was used. 
Since all YMs have locally bounded supports, we can always restrict our view to a finite ball $B_{R}=\left\{u \in \mathbb{R}^{d}:|u| \leqslant R\right\}$ such that $\mathrm{P}\left(B_{R}\right) \subset \mathrm{P}_{\mathrm{c}}\left(\mathbb{R}^{d}\right)$. From $[15$, theorem 6.3.3] we know that $\left(\mathrm{P}\left(B_{R}\right), \mathrm{d}_{\mathrm{W}}\right)$ is a complete metric space. It contains the set

$$
\left\{\sum_{1}^{n} a_{j} \delta_{u^{j}}: n \in \mathbb{N}, a_{j} \geqslant 0, \sum a_{j}=1, u^{j} \in B_{R}\right\}
$$

as dense subset. We will use the following helpful result.

LEMMA 2.2. The topology on $\mathrm{P}\left(B_{R}\right)$ induced by the Wasserstein distance is identical to the weak $k^{*}$ topology of $\mathrm{M}\left(B_{R}\right)$ restricted to $\mathrm{P}\left(B_{R}\right)$.

This is a direct consequence of the following general fact. Let $X$ be a separable Banach space such that the Banach space $Y$ is densely and compactly embedded into $X$. Then, the weak ${ }^{*}$ topology on the closed unit ball $\mathcal{B}$ in the dual space $X^{\prime}$ is identical to the topology induced by the norm $\|\cdot\|_{Y^{\prime}}$. To obtain lemma 2.2 , take $X=C^{0}\left(B_{R}\right)$ and $Y=C^{\mathrm{Lip}}\left(B_{R}\right)$.

Using the Wasserstein distance we define the Wasserstein metric on $\mathrm{YM}_{\mathrm{c}}\left(\Omega, \mathbb{R}^{d}\right)$ as follows

$$
\operatorname{dist}_{p}(\mu, \hat{\mu})=\left(\int_{x \in \Omega}\left(\mathrm{d}_{\mathrm{W}}(\mu(x), \hat{\mu}(x))\right)^{p} \mathrm{~d} x\right)^{1 / p} .
$$

Considering classical functions $f, g \in L^{\infty}(\Omega)$ as YMs we find $\operatorname{dist}_{p}\left(\delta_{f}, \delta_{g}\right)=\| f-$ $g \|_{L^{p}}$. Convergence in this metric immediately implies weak* convergence but not vice versa. In particular, it is more difficult to approximate a $\mathrm{YM} \nu$ in the metric $\operatorname{dist}_{p}(\cdot, \cdot)$ than in the weak* sense. We will need the following two approximation results.

LEMMA 2.3. Let $\Omega \subset \mathbb{R}^{m}$ be open and bounded and $\eta \in \mathrm{YM}_{\mathrm{c}}\left(\Omega, \mathbb{R}^{d}\right)$. Then, for each $\varepsilon>0$ there exists a continuous function $g: \Omega \times \mathbb{R}^{d} \rightarrow[0, \infty)$ such that $\operatorname{dist}_{1}(\eta, \gamma)<\varepsilon$ where the $Y M \gamma$ is given by $\langle\gamma(x), \psi\rangle=\int_{\mathbb{R}^{d}} g(x, u) \psi(u) \mathrm{d} u$.

Proof. Choose $R>0$ such that $\operatorname{sppt}(\eta(x)) \subset B_{R}$ for a.e. $x \in \Omega$. Since $x \mapsto \eta(x) \in$ $\mathrm{P}\left(B_{R}\right)$ is weak* measurable, it is also measurable as a mapping into the metric space $\left(\mathrm{P}\left(B_{R}\right), \mathrm{d}_{\mathrm{W}}\right)$ due to lemma 2.2. Hence,

$$
\tilde{\eta}_{\alpha}(x)=\left(\operatorname{vol}\left(B_{x, \alpha}\right)\right)^{-1} \int_{B_{x, \alpha}} \eta(y) \mathrm{d} y,
$$

where $B_{x, \alpha}=\{y \in \Omega:|x-y|<\alpha\}$, exists in the sense of $\mathrm{d}_{\mathrm{W}}$-convergence, lies in $\mathrm{P}\left(B_{R}\right)$, and satisfies $\lim _{\alpha \rightarrow 0} \tilde{\eta}_{\alpha}(x)=\eta(x)$ for a.e. $x \in \Omega$. Thus, we find $\alpha>0$ such that $\operatorname{dist}_{1}\left(\tilde{\eta}_{\alpha}, \eta\right)<\frac{1}{2} \varepsilon$.

For $\kappa \in(0,1)$ we define

$$
g_{\kappa}(x, v)=\left\langle\tilde{\eta}_{\alpha}(x), \Psi_{\kappa}(u-v)\right\rangle_{u \in B_{R}}, \quad \text { with } \Psi_{\kappa}(w)=\frac{c_{d}}{\kappa^{d+1}} \max \{\kappa-|w|, 0\}
$$

where $c_{d}$ is chosen such that $\int_{\mathbb{R}^{d}} \Psi_{\kappa} \mathrm{d} w=1$. Using $g_{\kappa}$ as a density we obtain the $\mathrm{YM} \gamma_{\kappa}$. With $\left|\Psi_{\kappa}(v)-\Psi_{\kappa}(w)\right| \leqslant c_{d} \kappa^{-d-1}|v-w|$ and continuity of $x \mapsto \tilde{\eta}_{\alpha}(x)$, we 
conclude continuity of $g_{\kappa}$ in $(x, u)$. Moreover,

$$
\begin{aligned}
\mathrm{d}_{\mathrm{W}}\left(\tilde{\eta}_{\alpha}(x), \gamma_{\kappa}(x)\right) & =\sup \left\{\left|\left\langle\tilde{\eta}_{\alpha}, \psi\right\rangle-\int_{B_{R}} g_{\kappa}(x, v) \psi(v) \mathrm{d} v\right|: \operatorname{Lip}(\psi) \leqslant 1\right\} \\
& =\sup \left\{\left|\left\langle\tilde{\eta}_{\alpha}(x), \psi-T_{\kappa} \psi\right\rangle\right|: \operatorname{Lip}(\psi) \leqslant 1\right\},
\end{aligned}
$$

where $T_{\kappa} \psi(u)=\int_{B_{R}} \Psi_{\kappa}(u-v) \psi(v) \mathrm{d} v$. With

$$
\begin{aligned}
\left|T_{\kappa} \psi(u)-\psi(u)\right| & =\left|\int \Psi_{\kappa}(u-v)(\psi(v)-\psi(u)) \mathrm{d} v\right| \\
& \leqslant \int \Psi_{\kappa}(u-v) \operatorname{Lip}(\psi)|u-v| \mathrm{d} v=\tilde{c}_{d} \kappa \operatorname{Lip}(\psi),
\end{aligned}
$$

we conclude that $\operatorname{dist}_{1}\left(\tilde{\eta}_{\alpha}, \gamma_{\kappa}\right) \leqslant \tilde{c}_{d} \kappa \operatorname{vol}(\Omega)$. Thus, we find $\kappa>0$ such that $\operatorname{dist}_{1}\left(\eta, \gamma_{\kappa}\right) \leqslant \operatorname{dist}_{1}\left(\eta, \tilde{\eta}_{\alpha}\right)+\operatorname{dist}_{1}\left(\tilde{\eta}_{\alpha}\right)<\varepsilon$.

The above results shows that YMs can be approximated by YMs having a density that depends continuously on $(x, u)$. The next result states that YMs can be approximated by limits of rapidly oscillating almost-periodic functions in the following sense. Let $V \in C(\mathbb{R} \times \mathbb{T}, \mathbb{R})$, with $\mathbb{T}=\mathbb{R} / \mathbb{Z}$, and $v^{n}(x)=V(x$, wnx $)$ with $\omega \neq 0$. Then,

$$
v^{n} \stackrel{\mathrm{YM}}{\longrightarrow} \eta, \quad \text { with }\langle\eta(x), \psi\rangle=\int_{\mathbb{T}} \psi(V(x, z)) \mathrm{d} z,
$$

see proposition 8.4 for this result and a generalization to higher dimension.

Proposition 2.4. For each $\nu \in \mathrm{YM}_{\mathbf{c}}((a, b), \mathbb{R})$ and $\varepsilon>0$, there exists $V \in$ $C([a, b] \times \mathbb{T}, \mathbb{R})$ such that $\operatorname{dist}_{1}(\nu, \eta)<\varepsilon$ where $\eta \in \mathrm{YM}_{\mathrm{c}}((a, b), \mathbb{R})$ is defined via $\langle\eta(x), \psi\rangle=\int_{\mathbb{T}} \psi(V(x, z)) \mathrm{d} z$.

Proof. With lemma 2.3, we find $g \in C([a, b] \times[-R, R],[0, \infty))$ such that

$$
\operatorname{dist}_{1}(\nu, \gamma)<\frac{1}{2} \varepsilon, \quad \text { where }\langle\gamma(x), \psi\rangle=\int g(x, v) \psi(v) \mathrm{d} v
$$

For $\kappa \in(0,1)$, let $g_{\kappa}(x, v)=\kappa /(2 R)+(1-\kappa) g(x, v)$, then the associated YM, $\gamma_{\kappa}$, satisfies

$$
\begin{aligned}
\mathrm{d}_{\mathrm{W}}\left(\gamma(x), \gamma_{\kappa}(x)\right) & \leqslant \sup \left\{\int_{|v| \leqslant R}[\kappa /(2 R)+\kappa g(x, v)]|\psi(v)-\psi(0)| \mathrm{d} v: \operatorname{Lip}(\psi) \leqslant 1\right\} \\
& \leqslant 2 \kappa R .
\end{aligned}
$$

We let $\eta=\gamma_{\kappa}$ and obtain $\operatorname{dist}_{1}(\nu, \eta)<\varepsilon$ by choosing $\kappa$ sufficiently small.

It remains to show that $\eta$ can be represented by a suitable function $V(x, z)$. To this end, use the distribution function $G(x, v)=\int_{-R}^{v} g_{\kappa}(x, w) \mathrm{d} w$, which satisfies $\partial_{v} G(x, v) \geqslant \kappa /(2 R)$ for $v \in(-R, R)$. Hence, the inverse, $H(x, \cdot)=G(x, \cdot)^{-1}$, exists and satisfies $H \in C([a, b] \times[0,1],[-R, R])$. The desired function $V$ is given by $V(x, z)=H(x,|2 z-1|)$ for $z \in[0,1]$, where $V(x, 0)=V(x, 1)$ allows us to take $z \in \mathbb{T}$. 


\section{Partial differential equations}

Before going to the general case we give some notations for the case of one single velocity, that is $C=c_{1} I$. The associated hyperbolic problem is

$$
\partial_{t} u+c_{1} \partial_{x} u=f(t, x, u), \quad u(0, x)=v(x) \in \mathbb{R}^{d} .
$$

Here we also allow $f$ to be a Caratheodory function (measurable in $(t, x)$ and continuous in $u$ ). If $f$ is Lipschitz continuous in $u$, the solution is given in the form $u(t, x)=\Phi\left(t, x-c_{1} t, v\left(x-c_{1} t\right)\right)$, where $\Phi$ is defined such that $\alpha(t)=\Phi\left(t, y, \alpha_{0}\right)$ is the unique solution of $\dot{\alpha}=f\left(t, y+c_{1} t, \alpha\right), \alpha(0)=\alpha_{0}$.

Recall that $\mu$ is called a YM solution of (3.1) if it is the YM limit of a sequence $\left(u^{n}\right)_{n}$ of (weak) solutions of (3.1) that is bounded in $L_{\mathrm{loc}}^{\infty}\left(\mathbb{R}^{2}, \mathbb{R}^{d}\right)$.

TheOREM 3.1. Every $Y M$ solution $\mu \in \mathrm{YM}_{\mathrm{c}}\left(\mathbb{R}^{2}, \mathbb{R}^{d}\right)$ of (3.1) satisfies

$$
\partial_{t} \mu+c_{1} \partial_{x} \mu+D_{u} \mu[f(t, x, u)]=0 .
$$

If $f$ is Lipschitz continuous, then every $Y M$ solution $(\mu, \nu)$ of the Cauchy problem (3.1) satisfies

$$
\mu(t, x)=\Phi\left(t, x-c_{1} t, \cdot\right)_{*} \nu\left(x-c_{1} t\right),
$$

where $\Phi(t, y, \alpha)$ is the flow mapping associated to the ODE $\dot{\alpha}=f\left(t, y+c_{1} t, \alpha\right)$.

Proof. To show the first assertion we use that for any $\psi \in C_{\mathrm{c}}^{1}\left(\mathbb{R}^{d}, \mathbb{R}\right)$ the function $w^{n}(t, x)=\psi\left(u^{n}(t, x)\right)$ is a weak solution of $\partial_{t} w^{n}+c_{1} \partial_{x} w^{n}=D_{u} \psi\left(u^{n}\right)\left[f\left(t, x, u^{n}\right)\right]$. This means that for every test function $\phi=\phi(t, x) \in C_{\mathrm{c}}^{1}\left(\mathbb{R}^{2}, \mathbb{R}\right)$,

$$
\int_{(t, x) \in \mathbb{R}^{2}}\left[\left(\partial_{t} \phi+c_{1} \partial_{x} \phi\right) \psi\left(u^{n}\right)+\phi D_{u} \psi\left(u^{n}\right)\left[f\left(t, x, u^{n}\right)\right]\right] \mathrm{d}(t, x)=0 .
$$

Using $u^{n} \stackrel{\text { YM }}{\longrightarrow} \mu$ we can go to the limit in each of the terms and obtain exactly (3.2).

The second assertion follows easily since $u^{n}(t, x)=\Phi\left(t, x-c_{1} t, v^{n}\left(x-c_{1} t\right)\right)$ is the form of the weak classical solution. As $\Phi$ is a Caratheodory function we find, by $(2.1)$, that $u^{n}(0, \cdot) \stackrel{\text { YM }}{\longrightarrow} \nu$ implies $\mu(t, x)=\Phi\left(t, x-c_{1} t\right)_{*} \nu\left(x-c_{1} t\right)$.

REMARK 3.2. The notion of measure-valued solutions, as introduced by DiPerna [5] and used in [6], is the same as our YM solutions. However, it is often used only in the following very weak sense. A similar discussion of these two notions is given in [4]. For a general (quasilinear) equation

$$
\partial_{t}[a(u)]+\partial_{x}[b(u)]=f(u),
$$

a YM $\mu$ is called a measure-valued solution if

$$
\partial_{t}\langle\mu(t, x, \mathrm{~d} u), a(u)\rangle_{\mathbb{R}^{d}}+\partial_{x}\langle\mu(t, x, \mathrm{~d} u), b(u)\rangle_{\mathbb{R}^{d}}=\langle\mu(t, x, \mathrm{~d} u), f(u)\rangle_{\mathbb{R}^{d}}
$$

holds in the sense of distributions. This is only a statement on the averages of the functions $a, b$ and $f$ with respect to the YM, whereas our definition is concerned with the full information on the measure. For instance,

$$
\mu(t, x)=\alpha(t, x) \delta_{-1}+\beta(t, x) \delta_{0}+(1-\alpha(t, x)-\beta(t, x)) \delta_{1}, \quad \alpha, \beta, 1-\alpha-\beta \geqslant 0,
$$


is a measure-valued solution of $\left(\partial_{t}+c \partial_{x}\right) u=0$ if and only if $\left(\partial_{t}+c \partial_{x}\right)[1-2 \alpha-\beta]=0$. For a YM solution we need more: $\left(\partial_{t}+c \partial_{x}\right) \alpha=\left(\partial_{t}+c \partial_{x}\right) \beta=0$.

One might hope that adding entropy conditions to $(3.5)$ restricts the set of measure-valued solutions. Here the entropy condition reads $\left(\partial_{t}+c \partial_{x}\right)\langle\mu, g\rangle \leqslant 0$ for all convex $g$, and for our $\mu$ this is equivalent to $\left(\partial_{t}+c \partial_{x}\right) \beta \geqslant 0$. Hence, the above notion of measure-valued solutions (even including entropy conditions) is much weaker than that of YM solutions used here. The former notion is mainly intended for problems where (3.5) is just an intermediate step, and subsequently it is shown that $\mu$ is in fact a point measure $\delta_{u(t, x)}$. Then, it is immediate that the function $u=u(t, x)$ is a solution of (3.4).

At first sight a generalization of $(3.2)$ for cases of different wave speeds $c_{j}$ might be

$$
\partial_{t} \mu+C \partial_{x} \mu+D_{u} \mu[f(u)]=0,
$$

which, of course, needs a suitable interpretation in the sense of distributions, namely

$$
\int_{(t, x) \in \mathbb{R}^{2}}\left\langle\mu(t, x, \mathrm{~d} u),\left(\partial_{t} \phi+C^{T} \partial_{x} \phi\right) \cdot \psi(u)+\phi \cdot D_{u} \psi(u)[f(u)]\right\rangle_{u \in \mathbb{R}^{d}} \mathrm{~d}(t, x)=0,
$$

for all test functions $\phi \in C_{\mathrm{c}}^{1}\left(\mathbb{R}^{2}, \mathbb{R}^{d}\right)$ and $\psi \in C_{\mathrm{c}}\left(\mathbb{R}^{d}, \mathbb{R}^{d}\right)$. However, this is far too much to ask for; in fact, it does not even allow for all classical solutions. This is easily seen by taking $d=2, c_{1} \neq c_{2}$ and $f \equiv 0$. Then, $u(t, x)=\left(\sin \left(x-c_{1} t\right), \sin \left(x-c_{2} t\right)\right)$ is a classical solution, but (3.8) is violated for $\mu(t, x)=\delta_{u(t, x)}$ and $\psi$ such that $\psi(u)=\left(u_{2}, 0\right)$ for $|u| \leqslant 2$. The problem is that the test functions $\psi$ should be such that $C D_{u} \psi(u)=D_{u} \psi(u) C$ for all $u$. Only under this condition we know that for any weak solution $u$ of (1.1) the function $w(t, x)=\psi(u(t, x))$ solves $\partial_{t} w+C \partial_{x} w=$ $D_{u} \psi(u(t, x)) f(t, x, u(t, x))$.

We now assume that the system matrix $C$ is diagonal with $C=\operatorname{diag}\left(c_{1}, \ldots, c_{d}\right)$ with all wave speeds $c_{j}$ different. Then $C D_{u} \psi(u)=D_{u} \psi(u) C$ can only hold for all $u$ if $\psi$ has the form $\left(\psi_{1}\left(u_{1}\right), \ldots, \psi_{d}\left(u_{d}\right)\right)$. The case of having some multiple wave speeds is completely analogous, if we take the corresponding component $u_{j}$ lying in $\mathbb{R}^{m_{j}}$ with associated marginal measures $\mu_{j} \in \mathrm{YM}_{\mathrm{c}}\left(\mathbb{R}^{2}, \mathbb{R}^{m_{j}}\right)$. For the sake of simple notations we restrict ourselves to $m_{j}=1$, but in the general case everything is the same, word for word. (That explains why we chose to prove theorems 3.1 and 5.1 in the vector-valued setting.)

The restriction to scalar test functions leads to a system of $d$ coupled equations, namely

$$
\int_{\mathbb{R}^{2}}\left\langle\mu(\mathrm{d} u),\left(\partial_{t} \phi+c_{j} \partial_{x} \phi\right) \psi\left(u_{j}\right)+\phi \psi^{\prime}\left(u_{j}\right) f_{j}(u)\right\rangle_{u \in \mathbb{R}^{d}} \mathrm{~d}(t, x)=0, \quad \text { for } j=1, \ldots, d,
$$

for all test functions $\phi \in C_{\mathrm{c}}^{1}\left(\mathbb{R}^{2}, \mathbb{R}\right)$ and $\psi \in C_{\mathrm{c}}^{1}(\mathbb{R}, \mathbb{R})$. Using the same argument as for the first assertion of theorem 3.1 we obtain the following result.

THEOREM 3.3. Every YM solution of (1.1) satisfies (3.9), respectively, (3.10) below. 
The main problem with system (3.9) is that it is generally not enough to determine the temporal evolution of YM solutions, even if suitable initial conditions are provided. In fact, (3.9) is no longer sufficient to determine the set of all YM solutions of (1.1) uniquely.

Using the marginal measures $\bar{\mu}=\left(\mu_{1}, \ldots, \mu_{d}\right)=\mathcal{M} \mu$ we can write (3.9) in a compact manner:

$$
\partial_{t} \mu_{j}+c_{j} \partial_{x} \mu_{j}=-\partial_{u_{j}}\left(\mathcal{Q}_{\nexists j}^{(\mu)}\left[f_{j}(\cdot)\right]\right), \quad j=1, \ldots, d,
$$

where $\mathcal{Q}_{\not \supset j}^{(\mu)}[g(\cdot)](t, x)$ is the measure on $\mathbb{R}$ defined by

$$
\psi \mapsto\left\langle\mu(t, x, \mathrm{~d} u), \psi\left(u_{j}\right) g(u)\right\rangle_{u \in \mathbb{R}^{d}},
$$

and thus is exactly the average of $g$ with respect to the conditional measure of $\mu$ under the condition $u_{j}$.

The formulation of (3.10) shows the obvious problem that the left-hand side only contains the marginals $\mu_{j}$, whereas on the right-hand side the full measure $\mu$ is needed that generally cannot be reconstructed from the vector of marginals $\bar{\mu}=\left(\mu_{1}, \ldots, \mu_{d}\right)$. Moreover, we must recall that (3.10) necessarily holds for any YM solution $\mu$ of (1.1). However, theorems 5.1 and 5.3 show that in cases with $d \geqslant 2$ the validity of (3.10) is not sufficient for $\mu$ being a YM solution.

Thus, we propose an alternative to the correct system (3.10). This is found by assuming that $\mu$ is the product measure $\otimes \mu_{j}$. Whence the closure problem of going from the marginals, $\bar{\mu}$, to the full measure, $\mu$, disappears. The arising system is called the non-resonance system, and the question arises how the solutions of this system are related to the correct solutions. The non-resonance system reads

$$
\partial_{t} \mu_{j}+c_{j} \partial_{x} \mu_{j}+\partial_{u_{j}} \mu_{j}\left[f_{j}^{(\bar{\mu})}\left(t, x, u_{j}\right)\right]=0, \quad j=1, \ldots, d,
$$

where the functions $f_{j}^{(\bar{\mu})}$ are defined via

$$
f_{j}^{(\bar{\mu})}\left(t, x, u_{j}\right)=\left\langle\otimes_{k \neq j} \mu_{k}\left(t, x, \mathrm{~d} u_{k}\right), f_{j}(u)\right\rangle_{u_{\nexists j} \in \mathbb{R}^{d-1}} .
$$

The function $f_{j}^{(\bar{\mu})}$ is thus the average of $f_{j}$ with respect to all variables except for $u_{j}$, and the following result holds by a simple comparison of the definitions of the terms.

LEMMA 3.4. If $\mu=\otimes_{j=1}^{d} \mu_{j}$, then $\mathcal{Q}_{\not j}^{(\mu)}\left[f_{j}(u)\right]=f_{j}^{(\bar{\mu})}\left(u_{j}\right) \mu_{j}$.

For general YM solutions $\mu$ this formula does not hold, see example 6.3.

The non-resonance system (3.11) governs the marginals $\bar{\mu}=\mathcal{M} \mu$, in the case when $\mu$ is a product measure (see also $[10,11]$ ). However, its importance reaches further, and we summarize some of it here.

(1) We can prove existence and uniqueness of solutions if initial conditions $\bar{\nu}$ are added. We can define a flow $\mathcal{S}_{t}$ on $\left(\mathrm{YM}_{\mathrm{c}}(\mathbb{R}, \mathbb{R})\right)^{d}$ such that $\bar{\mu}(t)=\mathcal{S}_{t}(\bar{\nu})$ (see $\S 4)$.

(2) Every solution of (3.11) defines a YM solution $\mu=\otimes_{1}^{d} \mu_{j}$ of (1.1), even if there are other YM solutions that are not in product form. This fact, which is proved in $\S 8$, cannot be deduced from (3.10) since this is only a necessary condition for a YM solution. 
(3) Under certain interaction conditions on $f$ (see (5.3)), the marginals $\bar{\mu}=\mathcal{M} \mu$ of every YM solution $\mu$ automatically satisfy the non-resonance system (3.11), even if $\mu$ itself is not a product measure.

We now make explicit in what sense a YM solution $\mu$ attains initial conditions.

THEOREM 3.5. Let $\mu$ be a YM solution of (1.1). Then for all $j=1, \ldots, d$ the mappings $t \mapsto \mu_{j}(t, \cdot) \in \mathrm{YM}_{\mathrm{c}}(\mathbb{R}, \mathbb{R})$ are weak $k^{*}$ continuous.

Proof. If $\mu$ is a YM solution then there exists a sequence $u^{n}$ of solutions such that $u^{n} \stackrel{\mathrm{YM}}{\longrightarrow} \mu$ and in particular $u_{j}^{n} \stackrel{\mathrm{YM}}{\longrightarrow} \mu_{j}$.

Take any test functions $\phi, \psi \in C_{0}^{1}(\mathbb{R}, \mathbb{R})$, then $\left(\partial_{t}+c_{j} \partial_{x}\right) u_{j}^{n}$ is essentially bounded over $n \in \mathbb{N}$ on the support of $\phi$. Denote this bound by $K$. Thus, we find

$$
\begin{aligned}
& \left|\int_{x \in \mathbb{R}} \phi(x) \psi\left(u_{j}^{n}(t, x)\right) \mathrm{d} x-\int_{x \in \mathbb{R}} \phi(x) \psi\left(u_{j}^{n}(t+\tau, x)\right) \mathrm{d} x\right| \\
& \leqslant\left|\int_{x \in \mathbb{R}} \phi(x)\left[\psi\left(u_{j}^{n}(t, x)\right)-\psi\left(u^{n}\left(t+\tau, x+c_{j} \tau\right)\right)\right] \mathrm{d} x\right| \\
& \quad+\left|\int_{y \in \mathbb{R}}\left[\phi\left(y-c_{j} \tau\right)-\phi(y)\right] \psi\left(u_{j}^{n}(t+\tau, y)\right) \mathrm{d} y\right| \\
& \leqslant\|\phi\|_{L^{1}}\left\|\psi^{\prime}\right\|_{L^{\infty}} K|\tau|+\left\|\phi^{\prime}\right\|_{L^{1}}\|\psi\|_{L^{\infty}}\left|c_{j} \tau\right| .
\end{aligned}
$$

Since the last term does not depend on $n$ we can go to the limit $n \rightarrow \infty$ in the first term and obtain $\int_{\mathbb{R}} \phi(x)\left\langle\mu_{j}(t+\tau, x), \psi\right\rangle \mathrm{d} x=\int_{\mathbb{R}} \phi(x)\left\langle\mu_{j}(t, x), \psi\right\rangle \mathrm{d} x+\mathcal{O}(|\tau|)$ for differentiable test functions. By density of $C^{1}$ in $C^{0}$ and boundedness of $t \mapsto$ $\int_{\mathbb{R}} \phi(x)\left\langle\mu_{j}(t, x), \psi\right\rangle \mathrm{d} x$, the continuity holds for all test functions.

This result allows us to prescribe initial conditions in the form $\mu_{j}(0, \cdot)=\nu_{j} \in$ $\mathrm{YM}_{\mathrm{c}}(\mathbb{R}, \mathbb{R})$. It is not clear whether a similar continuity result holds for the full measure $\mu$. Yet, the sequence $u^{n}(t, x)=(\cos (n(x+t)), \sin (n(x-t)))$ provides a simple example for a sequence such that

$$
u^{n} \stackrel{\mathrm{YM}}{\longrightarrow} \mu \in \mathrm{YM}_{\mathrm{c}}\left(\mathbb{R}^{2}, \mathbb{R}^{2}\right) \text { and } u^{n}(0, \cdot) \stackrel{\mathrm{YM}}{\longrightarrow} \nu \in \mathrm{YM}_{\mathrm{c}}\left(\mathbb{R}, \mathbb{R}^{2}\right),
$$

where $t \mapsto \mu(t, \cdot)$ is weak $^{*}$ continuous but $\mu(0, \cdot) \neq \nu$. In fact, $\mu$ is a product measure, which is independent of $(t, x)$, and $\nu$ is the $x$-independent measure of uniform distribution on the circle $u_{1}^{2}+u_{2}^{2}=1$.

\section{Existence and uniqueness}

We prove existence and uniqueness for the non-resonance system (3.11).

THEOREM 4.1. Assume that $f: \mathbb{R}^{d} \rightarrow \mathbb{R}^{d}$ is bounded and globally Lipschitz continuous $u$. Then, for any given $\bar{\nu} \in\left(\mathrm{YM}_{\mathrm{c}}(\mathbb{R}, \mathbb{R})\right)^{d}$, there exists a unique solution $\bar{\mu} \in\left(\mathrm{YM}_{\mathrm{c}}\left(\mathbb{R}^{2}, \mathbb{R}\right)\right)^{d}$ of system (3.11) satisfying the initial condition $\bar{\mu}(0, \cdot)=\bar{\nu}$.

For the proof we set up an iteration scheme of Banach-Picard type as is usually done for semilinear wave equations. However, this is not possible in the space of YM since the norm in the space of measures is much too strong. We therefore use an idea that is well known in the theory of transport equations of Vlasov 
type (see $[3]$ or $[9, \S 4.3]$ ). This idea says that iterations should better be done on the characteristic curves along which the mass is transported. For the present result we will in fact iterate the right-hand sides, $f_{j}^{(\bar{\mu})}$, in a suitable function space. If convergence to a limit $f_{j}^{*}$ occurs, then the desired solutions $\mu_{j}^{*}$ are found by theorem 3.1 .

Proof. To set up the iteration procedure, define the mapping $\bar{\mu}=\bar{S}(\bar{g})$ (the notion $\bar{g}$ indicates that $\bar{g}$ is a $d$-tuple of scalar functions of the form $\left.\left(g_{1}\left(u_{1}\right), \ldots, g_{d}\left(u_{d}\right)\right)^{\mathrm{T}}\right)$, which associates with $\bar{g}$ the unique solutions $\bar{\mu}$ of (3.11) with right-hand sides $g_{j}$ and initial conditions $\nu_{j}$. Moreover, we define $\bar{g}=\bar{R}(\bar{\mu})$ by averaging the fixed function $f$ according to (3.12).

We start by letting $\bar{\mu}^{(1)}(t, \cdot)=\bar{\nu}$, then $\bar{f}^{(n)}=\bar{R}\left(\bar{\mu}^{(n)}\right)$ and $\bar{\mu}^{(n+1)}=\bar{S}\left(\bar{f}^{(n)}\right)$ defines the iteration. Instead of studying the mapping $\bar{\mu}^{(n+1)}=\bar{S} \circ \bar{R}\left(\bar{\mu}^{(n)}\right)$ in the space of $Y M$, it is much more convenient to consider the mapping $\bar{f}^{(n+1)}=\bar{T}\left(\bar{f}^{(n)}\right)$ with $\bar{T}=\bar{R} \circ \bar{S}$, which is easier to handle since $d$ scalar functions are iterated.

We define a function space $Z$, such that $\bar{T}$ is well defined on $\bar{Z}=Z^{d}$ :

$$
\begin{aligned}
Z=\left\{g: \mathbb{R}^{2} \times \mathbb{R} \rightarrow\right. & \mathbb{R}: g \text { measurable, }|g(t, x, \alpha)| \leqslant C, \\
& \text { and }|g(t, x, \alpha)-g(t, x, \beta)| \leqslant L|\alpha-\beta| \text { for a.e. } t, x \text { and all } \alpha, \beta\},
\end{aligned}
$$

where $C$ and $L$ are the $L^{\infty}$-norm and the Lipschitz constant of $f$, respectively. The space $Z$ is a complete metric space (closed subset of a Banach space) when the norm

$$
\|g-h\|_{Z}=\sup \left\{|g(t, x, \alpha)-h(t, x, \alpha)| \mathrm{e}^{-3 L|t|}: t, x \in \mathbb{R}, \alpha \in \mathbb{R}\right\},
$$

is used.

For each $\bar{\mu} \in\left(\mathrm{YM}_{\mathrm{c}}\left(\mathbb{R}^{2}, \mathbb{R}\right)\right)^{d}$, the function $\bar{g}=\bar{R}(\bar{\mu})$ lies always in $\bar{Z}$, since averaging neither increases the $L^{\infty}$-bound nor the Lipschitz constant that are given from $f$. Moreover, for each $\bar{g} \in \bar{Z}$, the flow mappings $\Phi_{j}$ associated with (3.11) are globally well defined such that the mapping $\bar{S}: \bar{Z} \rightarrow\left(\mathrm{YM}_{\mathrm{c}}\left(\mathbb{R}^{2}, \mathbb{R}\right)\right)^{d}$ exists. Thus, $\bar{T}=\bar{R} \circ \bar{S}$ maps $\bar{Z}$ into itself. Our aim is to show that $\bar{T}$ is a contraction. For $\bar{g}, \bar{h} \in \bar{Z}$ we want to estimate $\bar{T}(\bar{g})-\bar{T}(\bar{h})$. To that end we solve for each $j$ and $y \in \mathbb{R}$ the ODEs

$$
\begin{aligned}
\dot{\alpha} & =g_{j}\left(t, y+c_{j} t, \alpha\right), & \alpha(0) & =\gamma_{0}, \\
\dot{\beta} & =h_{j}\left(t, y+c_{j} t, \beta\right), & \beta(0) & =\gamma_{0} .
\end{aligned}
$$

The associated flow maps are called $\Phi_{j}$ and $\Psi_{j}$, respectively. A Gronwall estimate gives

$$
\left|\Phi_{j}\left(t, y, \gamma_{0}\right)-\Psi_{j}\left(t, y, \gamma_{0}\right)\right|=|\alpha(t)-\beta(t)| \leqslant \frac{\mathrm{e}^{3 L|t|}}{2 L}\left\|g_{j}-h_{j}\right\|_{Z}
$$

since

$$
\left|g_{j}(t, z, \alpha)-h_{j}(t, z, \beta)\right| \leqslant L|\alpha-\beta|+\mathrm{e}^{3 L|t|}\left\|g_{j}-h_{j}\right\|_{Z} .
$$

The two measures solving (3.11) with initial condition $\bar{\nu}$ and nonlinearity $g$ and $h$ are denoted by $\mu^{g}$ and $\mu^{h}$, respectively, such that $\mu_{j}^{g}=\Phi_{j *} \nu_{j}$ and $\mu_{j}^{h}=\Psi_{j *} \nu_{j}$. Hence, $\hat{g}=\bar{T}(\bar{g})$ has the form

$$
\hat{g}_{j}\left(t, x, u_{j}\right)=\left\langle\otimes_{k \neq j} \nu_{k}\left(y, \mathrm{~d} w_{k}\right), f_{j}\left(\Phi_{1}\left(t, y, w_{1}\right), \ldots, u_{j}, \ldots, \Phi_{d}\left(t, y, w_{d}\right)\right)\right\rangle_{\mathbb{R}^{d-1}},
$$


where $y=x-c_{j} t$, and the analogous expression for $\hat{h}=\bar{T}(\bar{h})$ holds. Employing (4.1) and the Lipschitz continuity of $f_{j}$ in $u_{k}, k \neq j$, leads us to

$$
\left|\hat{g}_{j}\left(t, x, u_{j}\right)-\hat{h}_{j}\left(t, x, u_{j}\right)\right| \leqslant L \frac{\mathrm{e}^{3 L|t|}}{2 L}\left\|g_{j}-h_{j}\right\|_{Z},
$$

for a.e. $(t, x)$ and all $u_{j}$. But this is the desired result

$$
\|\bar{T}(\bar{g})-\bar{T}(\bar{h})\|_{Z} \leqslant \frac{1}{2}\|\bar{g}-\bar{h}\|_{Z} .
$$

The unique fixed point $\bar{f}^{*}$ defines a unique YM $\bar{\mu}^{*}=\bar{S}\left(\bar{f}^{*}\right)$, which is the solution of our problem.

Thus, we have obtained global existence and uniqueness of solutions for the nonresonance system (3.11). Together with the continuity result in theorem 3.5 we are able to define the flow mapping $\left(\mathcal{S}_{t}\right)_{t \in \mathbb{R}}$ associated with (3.11) by

$$
\mathcal{S}_{t}:\left\{\begin{aligned}
\left(\mathrm{YM}_{\mathrm{c}}(\mathbb{R}, \mathbb{R})\right)^{d} & \rightarrow\left(\mathrm{YM}_{\mathrm{c}}(\mathbb{R}, \mathbb{R})\right)^{d} \\
\bar{\nu} & \mapsto \bar{\mu}(t, \cdot) .
\end{aligned}\right.
$$

Then, $\mathcal{S}_{t}$ has the group property $\mathcal{S}_{t} \circ \mathcal{S}_{\tau}=\mathcal{S}_{t+\tau}$ for all $t, \tau \in \mathbb{R}$. In $\S 7$ we study the continuity properties of $\mathcal{S}_{t}$ with respect to the argument $\bar{\nu}$. From theorem 3.5 we know that the map $t \mapsto \mathcal{S}_{t}(\bar{\nu}) \in \mathrm{YM}_{\mathrm{c}}(\mathbb{R}, \mathbb{R})$ is weak* continuous; now we can say more.

Proposition 4.2. The mapping $t \mapsto \mu_{j}\left(t, x-c_{j} t, \cdot\right) \in \mathrm{M}(\mathbb{R})$ is continuous in the Wasserstein distance, that is $\mathrm{d}_{\mathrm{W}}\left(\mu_{j}\left(t, x-c_{j} t\right), \mu_{j}\left(s, x-c_{j} s\right)\right)=\mathcal{O}(|t-s|)$ for $t \rightarrow s$.

Proof. From $\left|f_{j}(u)\right| \leqslant M$ we deduce

$$
\left|\Phi_{j}\left(t, x-c_{j} t, u_{j}\right)-\Phi_{j}\left(s, x-c_{j} s, u_{j}\right)\right| \leqslant|t-s| M .
$$

Thus,

$$
\begin{aligned}
\mathrm{d}_{\mathrm{W}}\left(\mu_{j}\left(t, x-c_{j} t\right), \mu_{j}\left(s, x-c_{j} s\right)\right) \\
=\sup _{\operatorname{Lip}(\psi) \leqslant 1}\left|\left\langle\mu_{j}(0, x), \psi\left(\Phi_{j}\left(t, x-c_{j} t, \cdot\right)\right)-\psi\left(\Phi_{j}\left(s, x-c_{j} s, \cdot\right)\right)\right\rangle \leqslant M\right| t-s \mid,
\end{aligned}
$$

where $\langle\mu(0, x), g\rangle \leqslant\|g\|_{\infty}$ was used.

At the end of this section we provide an estimate on the variance $\operatorname{Var}\left(\mu_{j}\right)$ of the marginals $\mu_{j}$. For any measure $\mu \in \mathrm{M}(\mathbb{R})$ we have

$$
\operatorname{Var}(\mu)=\left\langle\mu, w^{2}\right\rangle_{w \in \mathbb{R}}-\left(\langle\mu, w\rangle_{w \in \mathbb{R}}\right)^{2}=\left\langle\mu(\mathrm{d} v),\left\langle\mu(\mathrm{d} w), \frac{1}{2}(v-w)^{2}\right\rangle_{w \in \mathbb{R}}\right\rangle_{v \in \mathbb{R}}
$$

With

$$
L_{j, j}=\sup \left\{\left|\partial_{u_{j}} f_{j}(u)\right|: u \in \mathbb{R}^{d}\right\},
$$

we obtain the following result.

Proposition 4.3. For each $j$ and $t \in \mathbb{R}$ we have the estimate

$$
\mathrm{e}^{-2 L_{j, j}|t|} \operatorname{Var}\left(\mu_{j}(0, x)\right) \leqslant \operatorname{Var}\left(\mu_{j}\left(t, x-c_{j} t\right)\right) \leqslant \mathrm{e}^{2 L_{j, j}|t|} \operatorname{Var}\left(\mu_{j}(0, x)\right) .
$$


Proof. We recall that $\mu_{j}(t)$ is just the pull back of $\nu_{j}$ with respect to the flow mapping $\Phi_{j}$, namely

$$
\mu_{j}(t, x)=\Phi_{j}\left(t, x-c_{j} t, \cdot\right)_{*} \nu_{j}\left(x-c_{j} t\right) .
$$

Hence,

$$
\begin{aligned}
\operatorname{Var}\left(\mu_{j}(t)\right) & =\left\langle\mu_{j}(t, \mathrm{~d} v),\left\langle\mu_{j}(t, \mathrm{~d} w), \frac{1}{2}(v-w)^{2}\right\rangle\right\rangle \\
& =\left\langle\nu_{j}(\mathrm{~d} v),\left\langle\nu_{j}(\mathrm{~d} w), \frac{1}{2}\left(\Phi_{j}(t, v)-\Phi_{j}(t, w)\right)^{2}\right\rangle\right\rangle,
\end{aligned}
$$

where the argument $x-c_{j} t$ was suppressed for notational convenience. Now the result follows from the Lipschitz continuity of $\Phi_{j}(t, \cdot)$ and $\Phi_{j}(t, \cdot)^{-1}$ with Lipschitz constant $\mathrm{e}^{L_{j, j}|t|}$.

As in [7], we say that $\mu_{j}$ has microstructure in the point $\left(t_{0}, x_{0}\right)$ if it lies in the support of the function $\operatorname{Var}\left(\mu_{j}(\cdot, \cdot)\right)$. The above proposition shows that microstructure is transported along characteristics and can neither be generated nor destroyed in finite time. This is of course due to our assumption that the oscillations in each characteristic direction are non-resonant.

\section{Convergence results}

We are now asking under what conditions the marginals $\bar{\mu}$ of a general YM solutions $\mu$ of (1.1) solve the non-resonance system (3.11). Some results of this section are well known (see, for example, $[10,21]$ ), however, we state and prove them for completeness. To this end we use the theory of compactness through compensation in the form of the div-curl lemma in $[12,17]$. The basic observation for our semilinear hyperbolic problem is that oscillatory behaviour of $u_{j}^{n}$ can only occur perpendicular to the characteristics given by $x=y+c_{j} t$. Thus, if we have just two different wave speeds, the oscillations occur in linearly independent directions and the YM limit has to be a product measure.

TheOREM 5.1. Consider $v^{n} \in L_{\mathrm{loc}}^{\infty}\left(\mathbb{R}^{2}, \mathbb{R}^{k}\right)$ and $w^{n} \in L_{\mathrm{loc}}^{\infty}\left(\mathbb{R}^{2}, \mathbb{R}^{m}\right)$ and assume that there exists $b, c \in \mathbb{R}$ such that the sequences $v^{n}, w^{n},\left(\partial_{t}+b \partial_{x}\right) v^{n}$ and $\left(\partial_{t}+\right.$ $\left.c \partial_{x}\right) w^{n}$ are bounded in $L_{\text {loc }}^{\infty}\left(\mathbb{R}^{2}\right)$. Moreover, assume

$$
v^{n} \stackrel{Y M}{\longrightarrow} \nu \in \mathrm{YM}_{\mathrm{c}}\left(\mathbb{R}^{2}, \mathbb{R}^{k}\right) \text { and } w^{n} \stackrel{Y M}{\longrightarrow} \omega \in \mathrm{YM}_{\mathrm{c}}\left(\mathbb{R}^{2}, \mathbb{R}^{m}\right) .
$$

Then, if $b \neq c$, we have

$$
\left(v^{n}, w^{n}\right) \stackrel{Y M}{\longrightarrow} \nu \otimes \omega \in \mathrm{YM}_{\mathrm{c}}\left(\mathbb{R}^{2}, \mathbb{R}^{k+m}\right) .
$$

Proof. The result is equivalent to

$$
\begin{aligned}
& \int_{\mathbb{R}^{2}} \phi(t, x) \psi_{1}\left(v^{n}(t, x)\right) \psi_{2}\left(w^{n}(t, x)\right) \mathrm{d}(t, x) \rightarrow \int_{\mathbb{R}^{2}} \phi(t, x) \Psi_{1}(t, x) \Psi_{2}(t, x) \mathrm{d}(t, x), \\
& \text { where } \Psi_{1}(t, x)=\left\langle\nu(t, x, \mathrm{~d} \tilde{v}), \psi_{1}(\tilde{v})\right\rangle_{\mathbb{R}^{k}} \text { and } \Psi_{2}(t, x)=\left\langle\omega(t, x, \mathrm{~d} \tilde{w}), \psi_{2}(\tilde{w})\right\rangle_{\mathbb{R}^{m}}
\end{aligned}
$$

for all test functions $\phi, \psi_{1}$ and $\psi_{2}$. The convergence of $v^{n}$ and $w^{n}$ towards $\nu$ and $\omega$, respectively, just means that $\psi_{1}\left(v^{n}\right) \stackrel{*}{\rightarrow} \Psi_{1}$ and $\psi_{2}\left(v^{n}\right) \stackrel{*}{\rightarrow} \Psi_{2}$. The desired result (5.1) is equivalent to having $\psi_{1}\left(v^{n}\right) \psi_{2}\left(w^{n}\right) \stackrel{*}{\rightarrow} \Psi_{1} \Psi_{2}$. We let

$$
g^{n}=\left(\psi_{1}\left(v^{n}\right), b \psi_{1}\left(v^{n}\right)\right)^{\mathbf{T}} \quad \text { and } \quad h^{n}=\left(c \psi_{2}\left(w^{n}\right),-\psi_{2}\left(w^{n}\right)\right)^{\mathrm{T}} \text {, }
$$


such that

$$
\operatorname{div} g^{n}=\left(\partial_{t}+b \partial_{x}\right) \psi_{1}\left(v^{n}\right)=D_{v} \psi_{1}\left(v^{n}\right)\left[\left(\partial_{t}+b \partial_{x}\right) v^{n}\right]
$$

and

$$
\operatorname{curl} h^{n}=D_{w} \psi_{2}\left(w^{n}\right)\left[\left(\partial_{t}+c \partial_{x}\right) w^{n}\right]
$$

are bounded in $L^{\infty}$. Now, the div-curl lemma asserts that the scalar product, $g^{n} \cdot h^{n}=(c-b) \psi_{1}\left(v^{n}\right) \psi_{2}\left(w^{n}\right)$, converges weak* to the scalar product of the weak ${ }^{*}$ limits of $g^{n}$ and $h^{n}$, namely $(c-b) \Psi_{1} \Psi_{2}$. This is the desired result.

Since for every weak solution $u^{n}$ that is bounded in $L_{\mathrm{loc}}^{\infty}$ the functions $\left(\partial_{j}+\right.$ $\left.c_{j} \partial_{x}\right) u_{j}^{n}$ are equal to $f_{j}\left(u^{n}(t, x)\right)$, and thus also bounded in $L_{\mathrm{loc}}^{\infty}$, theorem 5.1 states that for every YM solution $\mu$ of (1.1) the two-dimensional marginals $M_{\{j, k\}} \mu$ are, because of $c_{j} \neq c_{k}$, always product measures, namely

$$
M_{\{j, k\}} \mu=\mu_{j} \otimes \mu_{k} .
$$

This fact was found already in $[6,10,21]$. In [11] the generalization

$$
M_{\{j, k, l\}} \mu=\mu_{j} \otimes \mu_{k} \otimes \mu_{l},
$$

is shown for cases of generic non-constant wave speeds $c_{j}(t, x), c_{k}(t, x)$ and $c_{l}(t, x)$. Additionally, a generalization of the following theorem to 'triple interactions' is given there.

THEOREM 5.2. Assume that $f$ is continuous and that $\mu$ is a YM solution of (1.1). If all $c_{j}$ are different and if the functions $f_{j}$ satisfy the interaction condition

$$
f_{j}(u)=\sum_{k=1}^{d} g_{j, k}\left(u_{j}, u_{k}\right), \quad j=1, \ldots, d,
$$

with continuous functions $g_{j, k}$, then $\bar{\mu}=\mathcal{M} \mu \in\left(\mathrm{YM}_{\mathrm{C}}\left(\mathbb{R}^{2}, \mathbb{R}\right)\right)^{d}$ is a solution of (3.11).

Under the additional assumption that $f$ is Lipschitz continuous, the marginals $\bar{\mu}$ of any $Y M$ solution $\mu$ of (1.1) are uniquely determined by the initial conditions $\bar{\nu} \in\left(\mathrm{YM}_{\mathrm{c}}(\mathbb{R}, \mathbb{R})\right)^{d}$ and have the form $\bar{\mu}(t)=\mathcal{S}_{t}(\bar{\nu})$.

This result does not say that (5.3) implies $\mu=\otimes \mu_{j}$. The importance is that even without $\mu$ being a product measure we still can conclude that (3.11) is true. In particular, all linear systems satisfy (5.3) and, thus, can be solved explicitly (see $\S 6.1$ ). In $\S 6.2$ we present an example with $f \equiv 0$ such that (3.11) trivially holds but still $\mu$ is not a product measure. In $[11, \S 5.1]$, condition (5.3) is called 'propagation of compactness'.

Proof. Since (3.10) holds for every YM solution, the only thing we have to show is that, under the given assumptions, the identity

$$
\partial_{u_{j}} \mathcal{Q}_{\nexists j j}^{(\mu)}\left[f_{j}(u)\right]=\partial_{u_{j}} \mu_{j}\left[f_{j}^{(\bar{\mu})}\left(u_{j}\right)\right]
$$

holds (compare to lemma 3.4). Since $f_{j}=\sum_{k} g_{j, k}$ it suffices to show the identity for each term separately:

$$
\begin{aligned}
& \left\langle\mu(\mathrm{d} u), \psi^{\prime}\left(u_{j}\right) g_{j, k}\left(u_{j}, u_{k}\right)\right\rangle_{\mathbb{R}^{d}}=\left\langle\left(M_{\{j, k\}} \mu\right)\left(\mathrm{d}\left(u_{j}, u_{k}\right)\right), \psi^{\prime}\left(u_{j}\right) g_{j, k}\left(u_{j}, u_{k}\right)\right\rangle_{\mathbb{R}^{2}} \\
& =\left\langle\mu_{j}\left(\mathrm{~d} u_{j}\right), \psi^{\prime}\left(u_{j}\right)\left\langle\mu_{k}\left(\mathrm{~d} u_{k}\right), g_{j, k}\left(u_{j}, u_{k}\right)\right\rangle_{\mathbb{R}}\right\rangle_{\mathbb{R}}=\left\langle\mu_{j}\left(\mathrm{~d} u_{j}\right), \psi^{\prime}\left(u_{j}\right) g_{j, k}^{(\bar{\mu})}\left(u_{j}\right)\right\rangle_{\mathbb{R}},
\end{aligned}
$$


where we have used (5.2) for the second identity.

Since condition (5.3) is trivially satisfied for $d \leqslant 2$ we have proved that the flow mapping $\mathcal{S}_{t}$ of (4.2) describes the general YM solutions of (1.1). For dimension $d \geqslant 3$ no such description of all YM solutions exists (note however the generalizations in [11] to the case $\left.c_{j}(t, x)\right)$. Nevertheless, it is possible to give some non-trivial restrictions on YM solutions by using a tool that generalizes $\mathrm{YM}$ and is called $H$-measure in [22] and defect measure in [8]. We give a short introduction in Appendix A.

TheOREM 5.3. Let $\mu \in \mathrm{YM}_{\mathrm{c}}\left(\mathbb{R}^{2}, \mathbb{R}^{d}\right)$ be a YM solution of (1.1). Take any three $i_{m} \in\{1, \ldots, d\}$ such that $c_{i_{n}} \neq c_{i_{m}}$ for $n \neq m$. Then for all $\phi_{m} \in C_{0}^{0}(\mathbb{R}, \mathbb{R})$, $m=1,2,3$, and a.e. $(t, x)$ we have

$$
\left|\left\langle\mu, \prod_{m=1}^{3}\left\langle\phi_{m}\left(u_{i_{m}}\right)-E_{\phi_{m}}\right)\right\rangle\right| \leqslant \frac{1}{\sqrt{ } 2} \prod_{m=1}^{3}\left\langle\mu_{m},\left(\phi_{m}\left(u_{m}\right)-E_{\phi_{m}}\right)^{2}\right\rangle^{1 / 2}
$$

for a.e. $(t, x)$, where $E_{\phi_{m}}(t, x)=\left\langle\mu_{i_{m}}(t, x), \phi_{m}\left(u_{i_{m}}\right)\right\rangle$.

In the case of four components with $c_{i_{1}} \neq c_{i_{2}}$ and $c_{i_{3}} \neq c_{i_{4}}$ we have

$$
\left|\left\langle\mu, \prod_{m=1}^{4}\left(\phi_{m}\left(u_{i_{m}}\right)-E_{\phi_{m}}\right)\right\rangle\right| \leqslant \prod_{m=1}^{4}\left\langle\mu_{i_{m}},\left(\phi_{m}\left(u_{i_{m}}\right)-E_{\phi_{m}}\right)^{2}\right\rangle^{1 / 2} .
$$

These estimates follow from theorems A.2 and A.3, respectively, when we apply them to the sequences

$$
w_{i_{m}}^{n}=\phi_{m}\left(u_{i_{m}}^{n}\right)-E_{\phi_{m}}, \quad \text { where } u^{n} \stackrel{\mathrm{YM}}{\longrightarrow} \mu .
$$

Note that application of Hölder's inequality only gives

$$
\left|\left\langle\mu, \prod_{1}^{N}\left(\phi_{m}\left(u_{i_{m}}\right)-E_{\phi_{m}}\right)\right\rangle\right| \leqslant \prod_{1}^{N}\left\langle\mu_{i_{m}},\left(\phi_{m}\left(u_{i_{m}}\right)-E_{\phi_{m}}\right)^{N}\right\rangle^{1 / N}
$$

which has worse exponents for $N \geqslant 3$.

The interaction condition (5.3) can be reformulated for $f \in C^{2}$ by using

$$
K_{j, k, l}=\sup \left\{\left|\partial_{u_{k}} \partial_{u_{l}} f_{j}(u)\right|: u \in \mathbb{R}^{d}\right\} .
$$

Then (5.3) is equivalent to $K_{j, k, l}=0$ for $(k, l) \in R_{j}=\left\{(k, l) \in\{1, \ldots, d\}^{2}: k \neq l \neq\right.$ $j \neq k\}$. The importance of $K_{j, k, l}$ was known before (cf. [24]), since it enables us to control the strength of oscillations in the $j$-component. The strength of oscillation $\sigma_{j}$ for the sequence $u_{j}^{n}$ is defined as the variance of the marginal $\mu_{j}$ :

$$
\sigma_{j}(t, x)=\operatorname{Var}\left(\mu_{j}(t, x)\right)=\left\langle\mu_{j}(t, x, \mathrm{~d} v),\left(v-E_{j}\right)^{2}\right\rangle_{\mathbb{R}},
$$

with

$$
E_{j}(t, x)=\left\langle\mu_{j}(t, x, \mathrm{~d} v), v\right\rangle_{\mathbb{R}}
$$

Here $E_{j}$ is the expectation value of $\mu_{j}$ that is the weak* limit of $u_{j}^{n}$.

For the non-resonant case we provided a simple estimate in proposition 4.3. For the general case, there is a result due to Tartar [24] as follows. 
THEOREM 5.4. Let $j \in\{1, \ldots, d\}$ be given. Assume that all $K_{j, k, l},(k, l) \in R_{j}$, and $\alpha_{j}=\sup \left\{\partial_{u_{j}} f_{j}(u): u \in \mathbb{R}^{d}\right\}$ are finite and that $c_{k} \neq c_{j}$ for $k \neq j$. If $\mu$ is aYM solution of (1.1), then the following differential inequality holds:

$$
\left(\partial_{t}+c_{j} \partial_{x}\right) \sigma_{j} \leqslant 2 \alpha_{j} \sigma_{j}+2 \sum_{(k, l) \in R_{j}} K_{j, k, l}\left\langle\mu(t, x, \mathrm{~d} u),\left|u_{j}-E_{j}\right|\left|u_{k}-E_{k}\right|\left|u_{l}-E_{l}\right|\right\rangle_{\mathbb{R}^{d}}
$$

Proof. It suffices to consider the case $j=1$, since all coordinates are similar. In the weak form of

$$
\left(\partial_{t}+c_{1} \partial_{x}\right)\left(u_{1}^{n}\right)^{m}=m\left(u_{1}^{n}\right)^{m-1} f_{1}\left(u^{n}\right),
$$

we can pass to the limit by using the YM convergence and obtain the weak form of

$$
\left(\partial_{t}+c_{1} \partial_{x}\right) \sigma_{1}=2\left\langle\mu(\mathrm{d} u), u_{1} f_{1}(u)\right\rangle_{\mathbb{R}^{d}}-2 E_{1}\left\langle\mu(\mathrm{d} u), f_{1}(u)\right\rangle_{\mathbb{R}^{d}} .
$$

The right-hand side takes the form $2\left\langle\mu(\mathrm{d} u),\left(u_{1}-E_{1}\right)\left(f_{1}(u)-f_{1}(E)\right)\right\rangle_{\mathbb{R}^{d}}$. We estimate this expression by using an elementary representation formula holding for all $C^{2}$ functions,

$$
\begin{aligned}
& f_{1}\left(E_{1}, u_{2}, \ldots\right)-f_{1}(E) \\
& =\sum_{k=2}^{d} \int_{0}^{1} \partial_{u_{k}} f_{1}\left(E_{1}, u_{2}^{s}, \ldots, u_{d}^{s}\right) \mathrm{d} s\left(u_{k}-E_{k}\right) \\
& =\sum_{k=2}^{d} \int_{0}^{1} \partial_{u_{k}} f_{1}\left(E_{1}, \ldots, u_{k}^{s}, E_{k+1}, \ldots\right) \mathrm{d} s\left(u_{k}-E_{k}\right) \\
& \quad+2 \sum_{(k, l) \in R_{1}} \int_{0}^{1} \int_{0}^{1} \partial_{u_{l}} \partial_{u_{k}} f_{1}\left(E_{1}, u_{2}^{s t}, \ldots, u_{k}^{s}, u_{k+1}^{s t}, \ldots\right) s \mathrm{~d} t \mathrm{~d} s\left(u_{k}-E_{k}\right)\left(u_{l}-E_{l}\right)
\end{aligned}
$$

with the short-hand $u_{n}^{s}=u_{n}+s\left(E_{n}-u_{n}\right)$. The terms in the first sum are in fact equal to $f_{1}\left(E_{1}, \ldots, u_{k}, E_{k+1}, \ldots\right)-f_{1}(E)$ and theorem 5.1 can be applied giving

$$
\begin{aligned}
& \left\langle\mu(\mathrm{d} u),\left(u_{1}-E_{1}\right)\left[f_{1}\left(E_{1}, \ldots, u_{k}, E_{k+1}, \ldots\right)-f_{1}(E)\right]\right\rangle_{\mathbb{R}^{d}} \\
& \quad=\left\langle\mu_{1}\left(\mathrm{~d} u_{1}\right), u_{1}-E_{1}\right\rangle_{\mathbb{R}}\left\langle\mu_{k}\left(\mathrm{~d} u_{k}\right), f_{1}\left(E_{1}, \ldots, u_{k}, E_{k+1}, \ldots\right)-f_{1}(E)\right\rangle_{\mathbb{R}}=0 .
\end{aligned}
$$

Hence, taking care of the additional term $f(u)-f\left(E_{1}, u_{2}, \ldots\right)$, the right-hand side in (5.7) can be estimated from above by

$$
\left\langle\mu(\mathrm{d} u), 2 \alpha_{1}\left(u_{1}-E_{1}\right)^{2}+2\left|u_{1}-E_{1}\right| \sum_{(k, l) \in R_{1}} K_{1, k, l}\left|u_{k}-E_{k}\right|\left|u_{l}-E_{l}\right|\right\rangle_{\mathbb{R}^{d}} .
$$

This establishes the desired result.

Exploiting (5.4) we derive a differential inequality for the strength of oscillations.

COROLlaRY 5.5. Under the assumptions of theorem 5.4 we have

$$
\left(\partial_{t}+c_{j} \partial_{x}\right) \sigma_{j} \leqslant 2 \alpha_{j} \sigma_{j}+2 \sigma_{j}^{1 / 2} \sum_{(k, l) \in R_{j}} K_{j, k, l} \sigma_{k}^{1 / 2} \sigma_{l}^{1 / 2}
$$


Proof. Let $\left(i_{1}, i_{2}, i_{3}\right)=(j, k, l)$ and $\tilde{E}_{m}=\left\langle\mu_{i_{m}},\left|u_{i_{m}}-E_{i_{m}}\right|\right\rangle$, then $\tilde{E}_{m} \leqslant \sigma_{i_{m}}^{1 / 2}$ and

$$
\begin{aligned}
\left\langle\mu, \prod_{1}^{3}\left|u_{i_{m}}-E_{i_{m}}\right|\right\rangle & =\left\langle\mu, \prod_{1}^{3}\left(\left|u_{i_{m}}-E_{i_{m}}\right|-\tilde{E}_{m}\right)\right\rangle+\prod_{1}^{3} \tilde{E}_{m} \\
& \leqslant \frac{1}{\sqrt{ } 2} \prod_{1}^{3}\left[\left\langle\mu_{i_{m}}, \prod_{1}^{3}\left(\left|u_{i_{m}}-E_{i_{m}}\right|-\tilde{E}_{m}\right)^{2}\right\rangle\right]^{1 / 2}+\prod_{1}^{3} \tilde{E}_{m} \\
& =\frac{1}{\sqrt{ } 2} \prod_{1}^{3}\left[\sigma_{i_{m}}-\tilde{E}_{m}^{2}\right]^{1 / 2}+\prod_{1}^{3} \tilde{E}_{m} \\
& \leqslant \max \left\{\frac{1}{\sqrt{ } 2} \prod_{1}^{3}\left(1-\beta_{m}^{2}\right)^{1 / 2}+\prod_{1}^{3} \beta_{m}: \beta_{m} \in[0,1]\right\} \prod_{1}^{3} \sigma_{i_{m}}^{1 / 2} \\
& =\prod_{1}^{3} \sigma_{i_{m}}^{1 / 2} .
\end{aligned}
$$

Here, theorem 5.1 was used for the first equality and (5.4) for the first estimate.

If all $K_{j, k, l}=0$ for all $(k, l) \in R_{j}$, then the variable $u_{j}$ cannot develop oscillations if $\sigma_{j}$ is zero at $t=0$, even if nonlinear interactions take place through $f_{j}$. This is compatible with our existence theorem and the representation of the solution using the one-dimensional flow mapping $\Phi_{j}$ as in theorem 3.1. In fact, if condition (5.3) is satisfied, then we know that the marginals $\mu_{j}$ satisfy the non-resonance system (3.11), and thus estimate (4.3) holds.

If condition (5.3) is violated such that one $K_{j, k, l}$ is non-zero, then non-zero $\sigma_{k} \sigma_{l}$ can generate non-trivial $\sigma_{j}$. Note that $\sigma_{j} \equiv 0$ is just one solution of the differential inequality, but the right-hand side is not Lipschitz continuous such that the classical Gronwall estimate does not hold. The example in $\S 6.3$ provides such a case, namely $\sigma_{1}=\sigma_{3} \equiv \frac{1}{4}$ and $\sigma_{2}=\frac{1}{12} t^{2}$.

There are situations where estimate (5.8) is useful for problems not satisfying condition (5.3). In some applications one knows a priori that for certain $j$ the components $u_{j}^{n}$ do not oscillate but have a strong limit $u_{j}^{\infty}$. Let us assume that these components have indices $j=m+1, \ldots, d$. Then, we can relax the interaction condition (5.3) to

$$
f_{j}(u)=\sum_{k=1}^{m} g_{j, k}\left(u_{j}, u_{k}, u_{m+1}, \ldots, u_{d}\right), \quad j=1, \ldots, d .
$$

THEOREM 5.6. Assume that $f$ satisfies the relaxed interaction condition (5.9). Let $\mu$ be a $Y M$ solutions of (1.1) with $\mu_{j}(t, x)=\delta_{w_{j}(t, x)}$ for $j=m+1, \ldots, d$. Then, $\bar{\mu}=\mathcal{M} \mu$ is a solution of (3.11).

Under the additional assumption that $f$ is Lipschitz continuous, the marginals $\vec{\mu}$ of any YM solution $\mu$ of (1.1) are uniquely determined by (3.11) and the initial conditions $\bar{\nu} \in\left(\mathrm{YM}_{\mathrm{c}}(\mathbb{R}, \mathbb{R})\right)^{d}$ with $\nu_{j}=\delta_{w_{j}(x)}$ for $j=m+1, \ldots, d$.

The proof of this statement is an easy generalization of the above results, hence we omit the details. Notice that under the assumptions of the theorem in (5.8) each term in the sum over $R_{j}$ vanishes: either $K_{j, k, l}$ or $\sigma_{k} \sigma_{l}$ is equal to 0 . 
A typical application is the semilinear wave equation

$$
\partial_{t}^{2} w=\partial_{x}^{2} w+g\left(w, \partial_{t} w, \partial_{x} w\right), \quad w(0, x)=w_{0}, \quad \partial_{t} w(0, x)=w_{1}(x) .
$$

Letting $u=\left(\partial_{t} w+\partial_{x} w, \partial_{t} w-\partial_{x} w, w\right)^{\mathrm{T}}$ we obtain

$$
\partial_{t} u+\left(\begin{array}{ccc}
-1 & 0 & 0 \\
0 & 1 & 0 \\
0 & 0 & 0
\end{array}\right) \partial_{x} u=\left(\begin{array}{c}
h(u) \\
h(u) \\
\frac{1}{2}\left(u_{1}+u_{2}\right)
\end{array}\right), \quad u(0, x)=\left(\begin{array}{c}
w_{1}+\partial_{x} w_{0} \\
w_{1}-\partial_{x} w_{0} \\
w_{0}
\end{array}\right)
$$

where $h(u)=g\left(u_{3}, \frac{1}{2}\left(u_{1}+u_{2}\right), \frac{1}{2}\left(u_{1}-u_{2}\right)\right)$. For this system, a sequence of initial conditions needs $\partial_{x} w_{0}^{n}$ and $w_{1}^{n}$ bounded, hence the initial condition for $u_{3}$ is automatically convergent. Thus, $u_{3}$ is the non-oscillating component, and since the right-hand side in the $u_{3}$ equation is $\frac{1}{2}\left(u_{1}+u_{2}\right)$ the relaxed interaction condition (5.9) is satisfied for any $g$.

\section{Some examples}

\subsection{Linear coupled systems}

For the linear system (with $c_{j} \neq c_{k}$ )

$$
\left(\partial_{t}+c_{j} \partial_{x}\right) u_{j}=\alpha_{j} u_{j}+\sum_{k \neq j} a_{j, k} u_{k}, \quad j=1, \ldots, d,
$$

with initial conditions $\bar{\nu}$, there is a simple way to calculate the marginals $\bar{\mu}(t)=$ $\mathcal{S}_{t}(\bar{\nu})$ of any YM solution $\mu$. Since interaction condition (5.3) holds, the marginals $\bar{\mu}$ satisfy (3.11). With the mean value $E_{j}=\left\langle\mu_{j}, u_{j}\right\rangle$ we arrive at

$$
\left(\partial_{t}+c_{j} \partial_{x}\right) \mu_{j}+\partial_{u_{j}} \mu_{j}\left[\alpha_{j} u_{j}+\sum_{k \neq j} a_{j, k} E_{k}\right]=0, \quad \mu_{j}(0, \cdot)=\nu_{j} .
$$

Testing this equation with $\psi_{j}\left(u_{j}\right)=u_{j}$ we obtain the linear system

$$
\left(\partial_{t}+c_{j} \partial_{x}\right) E_{j}=\alpha_{j} E_{j}+\sum_{k \neq j} a_{j, k} E_{k}, \quad E_{j}(0, x)=\left\langle\nu_{j}(x), u_{j}\right\rangle,
$$

which is a classical linear hyperbolic system. It can be solved iteratively as in the proof of theorem 4.1. As soon as all $E_{k}$ are known, we let

$$
g_{j}(t, x)=\sum_{k \neq j} a_{j, k} E_{k}(t, x)
$$

and find

$$
\mu_{j}(t, x)=\Phi_{j}\left(t, x-c_{j} t\right)_{*} \nu_{j}\left(x-c_{j} t\right),
$$

where

$$
\Phi_{j}(t, y, w)=\mathrm{e}^{\alpha_{j} t} w+\int_{0}^{t} \mathrm{e}^{\alpha_{j}(t-s)} g_{j}\left(s, y-c_{j} s\right) \mathrm{d} s .
$$

Thus, each marginal $\mu_{j}$ is an affine linear transformation of the initial value on the given characteristic. Coupling only occurs on the level of the mean values. 


\subsection{Non-uniqueness in an uncoupled linear system}

We consider three uncoupled linear equations

$$
\left(\partial_{t}+c_{j} \partial_{x}\right) u_{j}=0, \quad \text { with } c_{1}<c_{2}=0<c_{3},
$$

having the solutions

$$
u_{j}^{n}(t, x)=H\left(\alpha_{j}\left(y_{j}\right) n y_{j}+\theta_{j}\left(y_{j}\right)\right), \quad \text { where } y_{j}=x-c_{j} t,
$$

where $H(s)=H(s+2)$ with $H(s)=1$ for $s \in[0,1)$ and $H(s)=0$ for $s \in[1,2)$. The functions $\theta_{j}$ are arbitrary functions in $L^{\infty}(\mathbb{R})$ whereas $\alpha_{j}$ is assumed to be positive and piecewise constant.

Since $H$ attains the values 0 and 1 with equal distribution, it is immediate that

$$
u_{j}^{n} \stackrel{\mathrm{YM}}{\longrightarrow} \mu_{j}=\frac{1}{2}\left(\delta_{0}+\delta_{1}\right),
$$

for all $(t, x)$ and $j=1,2,3$. The full $\mathrm{YM} \mu \in \mathrm{YM}_{\mathrm{c}}\left(\mathbb{R}^{2}, \mathbb{R}^{3}\right)$ is, in general, not independent of $(t, x)$, however, its support is concentrated in the finite set $\{0,1\}^{3}$. Knowing the marginals $\mu_{j}$ explicitly, and using the fact that the three two-dimensional marginals are product measures with equal mass in each of the four points (cf. (5.2)), we conclude that $\mu(t, x)$ is uniquely determined by the function

$$
\gamma(t, x)=\mu(t, x,\{(1,1,1)\}) .
$$

Obviously, $w^{n} \stackrel{*}{\rightarrow} \gamma$ where $w^{n}=u_{1}^{n} u_{2}^{n} u_{3}^{n}$. Our construction shows that it is possible to generate quite general functions $\gamma(t, x)$.

Given the above form of the solutions $u_{j}^{n}$ we can calculate $\gamma$ explicitly as follows. Let $\left(t_{0}, x_{0}\right)$ be a point such that $\alpha_{j}$ and $\theta_{j}$ are continuous in $x_{0}-c_{j} t_{0}$. Then,

$$
\frac{1}{\left|Q\left(\varepsilon, t_{0}, x_{0}\right)\right|} \int_{(t, x) \in Q\left(\varepsilon, t_{0}, x_{0}\right)} w^{n}(t, x) \mathrm{d}(t, x)
$$

where

$$
Q\left(\varepsilon, t_{0}, x_{0}\right)=\left\{(t, x):\left|x-x_{0}-c_{1}\left(t-t_{0}\right)\right|<\varepsilon,\left|x-x_{0}-c_{3}\left(t-t_{0}\right)\right|<\varepsilon\right\},
$$

can be approximated up to an error $\mathcal{O}(1)_{\varepsilon \rightarrow 0}$ (uniformly in $n$ ) by

$$
\begin{aligned}
\beta_{\varepsilon}^{n}\left(t_{0}, x_{0}\right)= & \frac{1}{4 \varepsilon^{2}} \int_{\left|y_{1}\right|,\left|y_{3}\right|<\varepsilon} H\left(\alpha_{1} n\left(z_{1}+y_{1}\right)+\theta_{1}\right) \\
& \times H\left(\alpha_{2} n\left(z_{2}+\frac{c_{3} y_{1}-c_{1} y_{3}}{c_{3}-c_{1}}\right)+\theta_{2}\right) H\left(\alpha_{3} n\left(z_{3}+y_{3}\right)+\theta_{3}\right) \mathrm{d}\left(y_{1}, y_{3}\right),
\end{aligned}
$$

where $\alpha_{j}$ and $\theta_{j}$ are evaluated at $z_{j}=x_{0}-c_{j} t_{0}$. Hence,

$$
\gamma\left(t_{0}, x_{0}\right)=\lim _{\varepsilon \rightarrow 0} \lim _{n \rightarrow \infty} \beta_{\varepsilon}^{n}\left(t_{0}, x_{0}\right),
$$

whenever the limit exists.

Proposition 6.1. The limit $\lim _{n \rightarrow \infty} \beta_{\varepsilon}^{n}$ exists and is independent of $\varepsilon$. Define

$$
\kappa_{1}\left(t_{0}, x_{0}\right)=\frac{c_{3} \alpha_{2}\left(x_{0}-c_{2} t_{0}\right)}{\left(c_{3}-c_{1}\right) \alpha_{1}\left(x_{0}-c_{1} t_{0}\right)}, \quad \kappa_{3}\left(t_{0}, x_{0}\right)=\frac{-c_{1} \alpha_{2}\left(x_{0}-c_{2} t_{0}\right)}{\left(c_{3}-c_{1}\right) \alpha_{3}\left(x_{0}-c_{3} t_{0}\right)},
$$


and the function $F: \mathbb{R} \rightarrow \mathbb{R}$ by $F(s)=\frac{1}{4}\left(s^{2}-s\right)$ for $s \in[0,1], F(-s)=F(s)$ and $F(s+2)=F(s)$ for $s \in \mathbb{R}$. Then, $\gamma\left(t_{0}, x_{0}\right)$ is given as follows. If $\kappa_{j}=p_{j} / q_{j}$ for $q_{j}, p_{j} \in \mathbb{N}$ such that $p_{j} / q_{j}$ is relatively prime and if $q_{1}, p_{1}, q_{3}$ and $p_{3}$ are odd, then

$$
\gamma\left(t_{0}, x_{0}\right)=\frac{1}{8}-\frac{r^{3}}{q_{1}^{2} p_{1} q_{3}^{2} p_{3}} F\left(q_{1} q_{3} \tilde{\theta} / r\right)
$$

where $r=\operatorname{gcd}\left(q_{1}, q_{2}\right)$ (greatest common divisor) and

$$
\tilde{\theta}=\theta_{2}-p_{1} \theta_{1} / q_{1}-p_{3} \theta_{3} / q_{3}
$$

In all other cases for $\left(\kappa_{1}, \kappa_{3}\right)$ we have $\gamma\left(t_{0}, x_{0}\right)=\frac{1}{8}$.

Proof. We first consider the case that either $\kappa_{1} \notin \mathbb{Q}$ or $\kappa_{3} \notin \mathbb{Q}$, then $\beta_{\varepsilon}^{n}\left(t_{0}, x_{0}\right) \rightarrow \frac{1}{8}$ for $n \rightarrow \infty$. This follows from the fact that at least in one of the integration directions $y_{j}$ the integrand is really quasi-periodic.

Now assume $\kappa_{j}=q_{j} / p_{j}$ relatively prime. The integrand of $\beta_{\varepsilon}^{n}$ is now periodic in $\left(y_{1}, y_{3}\right)$, and for $n \rightarrow \infty$ we find the limit

$$
\begin{aligned}
\frac{1}{4} \int_{\left(\xi_{1}, \xi_{3}\right) \in(0,2)^{2}} H\left(q_{1} \xi_{1}+\theta_{1}\right) H\left(p_{1} \xi_{1}+p_{3} \xi_{3}+\theta_{2}\right) H\left(q_{3} \xi_{3}+\theta_{3}\right) \mathrm{d}\left(\xi_{1}, \xi_{3}\right) \\
=\frac{1}{4} \int_{\xi_{3} \in(0,2)} W_{q_{1}, p_{1}}\left(p_{3} \xi_{3}+\tilde{\theta}\right) H\left(q_{3} \xi_{3}\right) \mathrm{d} \xi_{3},
\end{aligned}
$$

which is independent of $\varepsilon . W$ is found by integration with respect to $\xi_{1}$ :

$$
\begin{aligned}
W_{q_{1}, p_{1}}(s) & =\int_{\xi_{1} \in(0,2)} H\left(q_{1} \xi_{1}\right) H\left(s+p_{1} \xi_{1}\right) \mathrm{d} \xi_{1}=\sum_{k=0}^{q_{1}-1} \int_{2 k / q_{1}}^{(2 k+1) / q_{1}} H\left(s+p_{1} \xi\right) \mathrm{d} \xi \\
& =\frac{1}{2}+\sum_{k=0}^{q_{1}-1} \frac{1}{p_{1}}\left[F^{\prime}\left(s+(2 k+1) p_{1} / q_{1}\right)-F^{\prime}\left(s+2 k p_{1} / q_{1}\right)\right] \\
& =\frac{1}{2}-\frac{2\left(q_{1} p_{1} \bmod 2\right)}{q_{1} p_{1}} F^{\prime}\left(q_{1} s\right) .
\end{aligned}
$$

Here we used the relation $H(s)=\frac{1}{2}+F^{\prime \prime}(s)$. The last equality is easily deduced from

$$
W^{\prime}(y)=\frac{1}{p_{1}} \sum_{n=0}^{2 q_{1}-1}(-1)^{n+1} H\left(y+n p_{1} / q_{1}\right),
$$

which is identically 0 for even $q_{1} p_{1}$ and equal to $\left(2 / p_{1}\right)\left[\frac{1}{2}-H\left(q_{1} y\right)\right]$ for odd $q_{1} p_{1}$.

The integration with respect to $\xi_{3}$ yields

$$
\gamma=\frac{1}{4} \sum_{k=0}^{q_{3}-1} \int_{2 k / q_{3}}^{(2 k+1) / q_{3}} W_{q_{1}, p_{1}}\left(\tilde{\theta}+p_{3} \xi_{3}\right) \mathrm{d} \xi_{3}=\frac{1}{8}-\frac{q_{1} p_{1} \bmod 2}{2 q_{1} p_{1}} M\left(q_{1} \tilde{\theta}\right),
$$

with

$$
M(s)=\sum_{k=0}^{q_{3}-1} \int_{2 k / q_{3}}^{(2 k+1) / q_{3}} F^{\prime}\left(s+q_{1} p_{3} \xi_{3}\right) \mathrm{d} \xi_{3}
$$


Now, $M$ can be expressed as

$$
M(s)=\frac{1}{q_{1} p_{3}} \sum_{n=1}^{2 q_{3}}(-1)^{n+1} F\left(s+n q_{1} p_{3} / q_{3}\right) .
$$

If $q_{1}=r \tilde{q}_{1}$ and $q_{3}=r \tilde{q}_{3}$ with $r=\operatorname{gcd}\left(q_{1}, q_{3}\right)$ then $\tilde{q}_{1} p_{3} / \tilde{q}_{3}$ is relatively prime, and we obtain

$$
M^{\prime \prime}(s)=\frac{r}{q_{1} p_{3}} \sum_{n=1}^{2 \tilde{q}_{3}}(-1)^{n+1} F^{\prime \prime}\left(s+n \tilde{q}_{1} p_{3} / \tilde{q}_{3}\right)=\frac{-2 r\left(\tilde{q}_{1} p_{3} \tilde{q}_{3} \bmod 2\right)}{q_{1} p_{3}} F^{\prime \prime}\left(\tilde{q}_{3} s\right),
$$

implying $M(s) \equiv 0$ for even $\tilde{q}_{1} p_{3}$ and

$$
M(s)=\frac{-2 r}{q_{1} p_{3} \tilde{q}_{3}^{2}} F\left(\tilde{q}_{3} s\right),
$$

for odd $\tilde{q}_{1} p_{3}$. Putting everything together the result is established.

ConjeCture 6.2. For every YM solution $\mu$ of (6.1) with $\operatorname{sppt}(\mu(t, x)) \subset\{0,1\}^{3}$ the estimate $\left|\gamma(t, x)-\frac{1}{8}\right| \leqslant \frac{1}{16}$ holds for a.e. $(t, x) \in \mathbb{R}^{2}$.

From $|F(s)| \leqslant \frac{1}{16}$, we immediately conclude that all solutions constructed above satisfy the assertion in the conjecture. However, we were not able to prove the result for general YM solutions. The $H$-measure theory provides a non-trivial bound as well, namely $\left|\gamma(t, x)-\frac{1}{8}\right| \leqslant \sqrt{ } 2 / 16$, see (5.4).

We now restrict ourselves to the case $c_{1}=-1, c_{2}=0$ and $c_{3}=1$ for simplicity. Moreover, we assume $\alpha_{2} \equiv 2$ such that $\kappa_{j}(t, x)=1 / \alpha_{j}\left(x-c_{j} t\right)$. The functions $\alpha_{j}, j=1,3$, are chosen piecewise constant such that $\alpha_{j}(x)=1$ if $x \in(-1,1)$ and $\alpha_{j}(x)=2$ else. Hence, proposition 6.1 implies

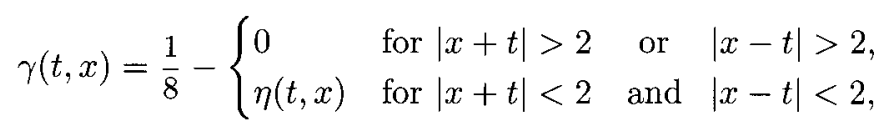

where

$$
\eta(t, x)=F\left(\theta_{1}(x+t)-\theta_{2}(x)+\theta_{3}(x-t)\right) .
$$

The functions $\theta_{j}$ are completely arbitrary.

Thus, we have constructed a YM solution $\mu \in \mathrm{YM}_{\mathrm{c}}\left(\mathbb{R}^{2}, \mathbb{R}^{3}\right)$ of $(6.1)$, which deviates from the product of its marginal measures $\mu_{j}=\frac{1}{2}\left(\delta_{0}+\delta_{1}\right)$ on an open bounded subset of $\mathbb{R}^{2}$. This implies that it is not possible to solve initial value problems uniquely.

In this case the YM solution $\mu(t, x)$ is supported on the eight points in $S=\{0,1\}^{3}$, such that it is possible to define the discrete information entropy

$$
\operatorname{Entr}(\mu)=-\sum_{u \in S} p_{u} \log \left(p_{u}\right)
$$

where $p_{u}=\mu(\{u\})$. Since $p_{u}$ either equals $\gamma$ or $\frac{1}{4}-\gamma$ depending on whether the number of $1 \mathrm{~s}$ in $u$ is odd or even, we have

$$
\operatorname{Entr}(\mu)=-4\left[\gamma \log \gamma+\left(\frac{1}{4}-\gamma\right) \log \left(\frac{1}{4}-\gamma\right)\right],
$$

which assumes its maximum value $\log 8$ for $\gamma=\frac{1}{8}$. Thus, the above example shows that the information entropy of a YM solution may increase as well as decrease with time. 


\subsection{A nonlinear example}

We treat a system of three equations that was already used in [10]. It shows that oscillations in some components can generate oscillations in other components. This provides an example where even the marginal measures $\mu_{j}$ are not uniquely determined.

Consider the system

$$
\left(\partial_{t}-\partial_{x}\right) u_{1}=0, \quad \partial_{t} u_{2}=2 u_{1} u_{3}, \quad\left(\partial_{t}+\partial_{x}\right) u_{3}=0,
$$

which has wave speeds 1,0 and +1 . We associate the YM initial conditions

$$
\mu_{1}(0, x)=\mu_{3}(0, x)=\frac{1}{2}\left(\delta_{0}+\delta_{1}\right), \quad \mu_{2}(0, x)=\delta_{0} .
$$

There is a unique product-measure solution $\mu=\mu_{1} \otimes \mu_{2} \otimes \mu_{3}$ (see theorem 8.1) solving the non-resonance system, which is given explicitly in (7.1) below. Since the first and third equations are decoupled we have $\mu_{j}(t, x)=\frac{1}{2}\left(\delta_{0}+\delta_{1}\right)$ for all $(t, x)$ and $j=1,3$. Averaging the right-hand side of the second equation according to $(3.12)$ gives the constant $\frac{1}{2}$. Thus, the $u_{2}$-component has the point measure $\mu_{2}(t, x)=\delta_{t / 2}$. This product-measure solution is also easily obtained by considering the sequence of solutions $u^{n}$ with initial conditions

$$
u_{1}^{n}(0, x)=H(n x), \quad u_{3}^{n}(0, x)=H(\omega n x), \quad u_{3}^{n}(0, x)=0,
$$

where $H$ is defined directly after $(6.2)$ and $\omega=2$ or $\omega \notin \mathbb{Q}$.

Since interaction condition (5.3) does not hold, we can expect more general solutions. We consider the sequence $\hat{u}^{n}$ of solutions given by the initial values in (6.4) but now with $\omega=1$. Integrating along the characteristic for $\hat{u}_{2}^{n}$, the integrand is periodic, and we find

$$
\hat{u}_{2}^{n}(t, x)=\int_{0}^{t} 2 H(n(x-s)) H(n(x+s)) \mathrm{d} s=t G(x n)+R(t, x, n),
$$

with $G(s)=G(s+1)=G(-s)$ and $G(s)=2 s$ for $s \in\left[0, \frac{1}{2}\right]$ and $R(t, x, n)=$ $\mathcal{O}(1 / n)_{n \rightarrow \infty}$ uniformly in $(t, x) \in \mathbb{R}^{2}$. The marginal measure $\mu_{2}(t, x)$ is distributed uniformly between $u_{2}=0$ and $u_{2}=t$. For $t>0$ the joint measure $\mu(t, x)$ has its support concentrated on four line segments in $\mathbb{R}^{3}$, namely $\operatorname{sppt}(\mu(t, x))=\left\{u \in \mathbb{R}^{3}\right.$ : $\left.u_{1}, u_{3} \in\{0,1\}, u_{2} \in[0, t]\right\}$. A geometric consideration of the periodic functions $H(n(x \pm t))$ and $t G(n x)$ gives

$$
\begin{aligned}
\langle\mu(t, x), \psi\rangle_{\mathbb{R}^{3}}=\int_{0}^{t}\{\psi(0, v, 0)+\psi(1, v, 1)\} & \frac{v}{2 t^{2}} \mathrm{~d} v \\
& +\int_{0}^{t}\{\psi(1, v, 0)+\psi(0, v, 1)\} \frac{t-v}{2 t^{2}} \mathrm{~d} v
\end{aligned}
$$

We now show by explicit calculation that $\mu$, defined by $(6.5)$, solves $(3.10)$, i.e.

$$
\left(\partial_{t}-\partial_{x}\right) \mu_{1}=0, \quad \partial_{t} \mu_{2}+\partial_{u_{2}} \eta_{2}=0, \quad\left(\partial_{t}+\partial_{x}\right) \mu_{3}=0,
$$

where

$$
\eta_{2}(t, x)=\mathcal{Q}_{\not 2}^{(\mu)}\left[2 u_{1} u_{3}\right](t, x) \in \mathrm{M}(\mathbb{R}),
$$

can be calculated explicitly from (6.5) to give

$$
\left\langle\eta_{2}, \psi\right\rangle=\int_{0}^{t} \psi(v) v / t^{2} \mathrm{~d} v
$$


cf. the definition of $\mathcal{Q}$ after (3.10). The first and third equations are trivial since $\mu_{1}=\mu_{3}=\frac{1}{2}\left(\delta_{0}+\delta_{1}\right)$, which is independent of $(t, x)$. The second equation takes the form

$$
\int_{\mathbb{R}^{2}}\left[\left\langle\mu_{2}(t, x), \partial_{t} \phi \psi\right\rangle+\left\langle\eta_{2}, \phi \psi^{\prime}\right\rangle\right] \mathrm{d}(t, x)=0,
$$

for every $\phi \in C_{\mathrm{c}}^{1}\left(\mathbb{R}^{2}, \mathbb{R}\right)$ and $\psi \in C_{\mathrm{c}}^{1}(\mathbb{R}, \mathbb{R})$. Employing

$$
\left\langle\mu_{2}, \psi\right\rangle=\int_{0}^{t} \frac{\psi(v)}{t} \mathrm{~d} v
$$

we obtain the desired result

$$
\begin{aligned}
\int_{\mathbb{R}^{2}}\left(\partial_{t} \phi \int_{0}^{t}\right. & \left.\frac{\psi(s)}{t} \mathrm{~d} s+\phi \int_{0}^{t} \frac{\psi^{\prime}(s) s}{2 t^{2}} \mathrm{~d} s\right) \mathrm{d}(t, x) \\
= & \int_{\mathbb{R}^{2}}\left(\phi\left\{\int_{0}^{t} \frac{\psi(s)}{t^{2}} \mathrm{~d} s-\frac{\psi(t)}{t}\right\}+\phi\left\{\frac{\psi(t)}{t}-\int_{0}^{t} \frac{\psi(s)}{t^{2}} \mathrm{~d} s\right\}\right) \mathrm{d}(t, x)=0 .
\end{aligned}
$$

Here we have $\eta_{2}=\left(u_{2} / t\right) \mu_{2} \neq f_{2}^{(\bar{\mu})}\left(u_{2}\right) \mu_{2}=\frac{1}{2} \mu_{2}$, which contrasts nicely with the result of lemma 3.4 since $\mu$ is not a product measure.

For this solution the oscillation strengths are $\sigma_{1}=\sigma_{3}=\frac{1}{4}$ and $\sigma_{2}=\frac{1}{12} t^{2}$. With $\alpha_{2}=0$ and $K_{2,1,3}=2$ the differential inequality (5.8) can be justified, namely

$$
\frac{1}{6} t=\left(\partial_{t}+0 \partial_{x}\right) \sigma_{2} \leqslant 0 \sigma_{2}+4\left(\sigma_{1} \sigma_{2} \sigma_{3}\right)^{1 / 2}=|t| / \sqrt{12} .
$$

\section{Continuity properties of $\mathcal{S}_{t}$}

In this section we study the continuity properties of the flow $\mathcal{S}_{t}$ defined in (4.2) that defines, via $\bar{\mu}(t)=\mathcal{S}_{t}(\bar{\nu})$, the solutions of the non-resonance system (3.11). An important observation is that continuity with respect to the weak* topology does not hold without further assumptions. Consider again system (6.3) treated above. The associated non-resonance system is given by

$$
\partial_{t} \mu_{1}-\partial_{x} \mu_{1}=0, \quad \partial_{t} \mu_{2}+\partial_{u_{2}} \mu_{2}\left[2\left\langle\mu_{1}, u_{1}\right\rangle\left\langle\mu_{3}, u_{3}\right\rangle\right]=0, \quad \partial_{t} \mu_{3}+\partial_{x} \mu_{3}=0 .
$$

The solution $\bar{\mu}^{\infty}(t)=\mathcal{S}_{t}\left(\bar{\nu}^{\infty}\right)$ with initial conditions

$$
\nu_{1}^{\infty}=\nu_{3}^{\infty}=\frac{1}{2}\left(\delta_{0}+\delta_{1}\right) \quad \text { and } \quad \nu_{2}^{\infty}=\delta_{0},
$$

is given by

$$
\mu_{1}^{\infty}(t, x)=\mu_{3}^{\infty}(t, x)=\frac{1}{2}\left(\delta_{0}+\delta_{1}\right) \quad \text { and } \quad \mu_{2}^{\infty}(t, x)=\delta_{t / 2} .
$$

Moreover, the classical solutions $\hat{u}^{n}$ given in $\S 6.3$ also define solutions $\bar{\mu}^{n}=\mathcal{S}_{t}\left(\bar{\nu}^{n}\right)$ of (7.1) via $\mu_{j}^{n}(t, x)=\delta_{\hat{u}_{j}^{n}(t, x)}$. These solutions satisfy

$$
\bar{\nu}^{n} \stackrel{*}{\rightarrow} \bar{\nu}^{\infty} \quad \text { and } \quad \bar{\mu}^{n} \stackrel{*}{\rightarrow} \bar{\mu}^{*}(t),
$$

for $n \rightarrow \infty$, where $\mu_{j}^{*}=\mu_{j}^{\infty}$ for $j=1,3$ but

$$
\left\langle\mu_{2}^{*}, \psi\right\rangle=\frac{1}{t} \int_{0}^{t} \psi\left(u_{2}\right) \mathrm{d} u_{2} .
$$

Hence, $\mu_{2}^{*}(t) \neq \mu_{2}^{\infty}(t)$ for $t \neq 0$, and $\mathcal{S}_{t}$ is not weak ${ }^{*}$ continuous. 
Thus, it is not surprising that our positive result concerning the weak* continuity involves the interaction condition (5.3).

THEOREM 7.1. Assume that $f$ is globally Lipschitz continuous and satisfies the interaction condition (5.3). Then, for each $t \in \mathbb{R}$ the flow mapping $\mathcal{S}_{t}$ is weak ${ }^{*}$ continuous from $\left(\mathrm{YM}_{\mathrm{c}}(\mathbb{R}, \mathbb{R})\right)^{d}$ into itself.

Proof. We have to show that $\bar{\nu}^{n} \stackrel{*}{\rightarrow} \bar{\nu}^{\infty}$ implies

$$
\bar{\mu}^{n}(t)=\mathcal{S}_{t}\left(\bar{\nu}^{n}\right) \stackrel{*}{\rightarrow} \bar{\mu}^{\infty}(t)=\mathcal{S}_{t}\left(\bar{\nu}^{\infty}\right) .
$$

We use theorem 5.2 (involving the interaction condition) and the fact that the set of all YM solutions of (1.1) is weak* closed.

To each $\bar{\nu}^{n}$ we can associate a YM solution, $\mu^{n} \in \mathrm{YM}_{\mathrm{c}}\left(\mathbb{R}^{2}, \mathbb{R}^{d}\right)$, such that $\mathcal{M} \mu^{n}(0, \cdot)=\bar{\nu}^{n}$. By theorem 5.2 we then know that $\mathcal{M} \mu^{n}=\bar{\mu}^{n}$. On the other hand, after extracting a subsequence if necessary, there is $\eta \in \mathrm{YM}_{\mathbf{c}}\left(\mathbb{R}^{2}, \mathbb{R}^{d}\right)$ with $\mu^{n} \stackrel{*}{\rightarrow} \eta$. Since $\eta$ also is a YM solution we conclude again that $\bar{\eta}=\mathcal{M} \eta$ has the form $\bar{\eta}(t)=\mathcal{S}_{t}(\bar{\eta}(0))$. It remains to show $\bar{\mu}^{n}(t) \stackrel{*}{\rightarrow} \bar{\eta}(t)$ in $\left(\mathrm{YM}_{\mathrm{c}}(\mathbb{R}, \mathbb{R})\right)^{d}$ and $\bar{\eta}(0)=\bar{\nu}^{\infty}$.

We choose a function $\phi \in C_{0}^{1}(\mathbb{R}, \mathbb{R})$ with $\phi \geqslant 0$ and $\int_{\mathbb{R}} \phi(t) \mathrm{d} t=1$. As in the proof of theorem 3.5 we obtain the following estimate: for any $\varphi, \psi \in C_{0}^{1}(\mathbb{R}, \mathbb{R})$ we have

$$
\left|\int_{\mathbb{R}^{2}} \frac{1}{\varepsilon} \phi(\varepsilon(s-t)) \varphi(x)\left\langle\mu_{j}(s, x), \psi\right\rangle \mathrm{d}(s, x)-\int_{\mathbb{R}} \varphi(x)\left\langle\mu_{j}(t, x), \psi\right\rangle \mathrm{d} x\right| \leqslant C \varepsilon,
$$

for any marginal measure $\mu_{j}$ of any YM solution $\mu$ of (1.1). Here $C$ depends only on the $L_{\mathrm{loc}}^{\infty}$ bounds for $\operatorname{diam}[\operatorname{sppt}(\mu(\cdot))]$, and on the test functions. Applying (7.2) to $\mu_{j}^{n}$ and $\eta_{j}$ and taking the difference gives

$$
\begin{aligned}
& \left|\int_{\mathbb{R}} \varphi(x)\left\langle\mu_{j}^{n}(t, x)-\eta_{j}(t, x), \psi\right\rangle \mathrm{d} x\right| \\
& \leqslant 2 C \varepsilon+\left|\int_{\mathbb{R}^{2}} \frac{1}{\varepsilon} \phi(\varepsilon(s-t)) \varphi(x)\left\langle\mu_{j}^{n}(s, x)-\eta_{j}(s, x), \psi\right\rangle \mathrm{d}(s, x)\right| .
\end{aligned}
$$

With $\mu_{j}^{n} \stackrel{*}{\rightarrow} \eta_{j}$ in $\mathrm{YM}_{\mathrm{c}}\left(\mathbb{R}^{2}, \mathbb{R}\right)$ we conclude $\mu_{j}^{n}(t, \cdot) \stackrel{*}{\rightarrow} \eta_{j}(t, \cdot)$ in $\mathrm{YM}_{\mathrm{c}}(\mathbb{R}, \mathbb{R})$ for each $t$. Moreover, since $\mu_{j}^{n}(0, \cdot)=\nu_{j}^{n}$, we conclude that $\nu_{j}^{\infty}=\eta_{j}(0, \cdot)$.

Next we establish continuity properties of $\mathcal{S}_{t}$ in the stronger topology defined by the Wasserstein metric. This approximation result will be useful in the next section. For this part we do not need any interaction condition on the nonlinearity $f(u)$.

Our aim is to compare the solutions $\bar{\mu}(t)=\mathcal{S}_{t}(\bar{\nu})$ and $\bar{\eta}(t)=\mathcal{S}_{t}(\bar{\kappa})$ if the distance between $\bar{\nu}$ and $\bar{\kappa}$ is controlled. We recall the definition of the Wasserstein distance

$$
\mathrm{d}_{\mathrm{W}}(\mu, \hat{\mu})=\sup \left\{|\langle\mu, \psi\rangle-\langle\hat{\mu}, \psi\rangle|: \psi \in C^{1}\left(\mathbb{R}^{m}, \mathbb{R}\right),\left\|\psi^{\prime}\right\|_{L^{\infty}} \leqslant 1\right\},
$$

for $\mu, \hat{\mu} \in \mathrm{P}_{\mathrm{c}}\left(\mathbb{R}^{m}\right)$. We define the functions

$$
a_{j}(t, x)=\mathrm{d}_{\mathrm{W}}\left(\mu_{j}(t, x), \eta_{j}(t, x)\right),
$$

and show that these functions can be controlled by their values at $t=0$.

THEOREM 7.2. Let $L_{j, k}=\sup \left\{\left|\partial_{u_{k}} f_{j}(u)\right|: u \in \mathbb{R}^{d}\right\}$. Then, for all pairs $(\bar{\mu}, \bar{\eta})$ of solutions of (3.11), the positive functions $a_{j} \in L_{\text {loc }}^{\infty}\left(\mathbb{R}^{2}, \mathbb{R}\right)$ defined above satisfy the 
inequality

$a_{j}(t, x) \leqslant \mathrm{e}^{L_{j, j}|t|} a_{j}\left(0, x-c_{j} t\right)+\int_{0}^{t} \mathrm{e}^{L_{j, j}(t-s)} D_{j}\left(s, x-c_{j}(t-s)\right) \mathrm{d} s, \quad j=1, \ldots, d$,

where $D_{j}(t, x)=\sum_{k \neq j} L_{j, k} a_{k}(t, x)$.

Proof. The solutions $\bar{\mu}$ and $\bar{\eta}$ are defined via the averaged functions

$$
g_{j}\left(t, x, u_{j}\right)=f_{j}^{(\bar{\mu})}\left(t, x, u_{j}\right) \quad \text { and } \quad h_{j}\left(t, x, u_{j}\right)=f_{j}^{(\bar{\eta})}\left(t, x, u_{j}\right)
$$

Using lemma 2.1 we find the estimate

$$
\begin{aligned}
\left|g_{j}\left(t, x, u_{j}\right)-h_{j}\left(t, x, u_{j}\right)\right| & \\
& =\left|\left\langle\otimes_{k \neq j} \mu_{j}\left(t, x, \mathrm{~d} u_{k}\right)-\otimes_{k \neq j} \eta_{j}\left(t, x, \mathrm{~d} u_{k}\right), f_{j}(u)\right\rangle\right| \leqslant D_{j}(t, x) .
\end{aligned}
$$

The flow mappings $\Phi_{j}$ and $\Psi_{j}$ are defined via $f_{j}$ and $g_{j}$, respectively, such that $\mu_{j}\left(t, y+c_{j} t\right)=\Phi_{j}(t, y)_{*} \mu_{j}(0, y)$ and similarly for $\eta_{j}$. Both mappings are Lipschitz continuous with constant $\mathrm{e}^{L_{j, j}|t|}$. We find

$$
\begin{aligned}
a_{j}\left(t, y+c_{j} t\right)= & \sup \left\{\left\langle\mu_{j}(0, y), \psi\left(\Phi_{j}(t, y, \cdot)\right)\right\rangle-\left\langle\eta_{j}(0, y), \psi\left(\Psi_{j}(t, y, \cdot)\right)\right\rangle: \operatorname{Lip}(\psi) \leqslant 1\right\} \\
\leqslant & \operatorname{Lip}\left(\Phi_{j}(t, y, \cdot)\right) \mathrm{d}_{\mathrm{W}}\left(\mu_{j}(0, y), \eta_{j}(0, y)\right) \\
& \quad+\sup \left\{\left\langle\eta_{j}(0, y), \psi\left(\Phi_{j}(t, y, \cdot)\right)-\psi\left(\Psi_{j}(t, y, \cdot)\right)\right\rangle: \operatorname{Lip}(\psi) \leqslant 1\right\} \\
\leqslant & \mathrm{e}^{L_{j, j}|t|} a_{j}(0, y)+\sup \left\{\left|\Phi_{j}(t, y, \xi)-\Psi_{j}(t, y, \xi)\right|: \xi \in \mathbb{R}\right\} .
\end{aligned}
$$

The estimate between the flow mappings is obtained from the estimate for $g_{j}-h_{j}$. Let

$$
\alpha(t)=\Phi(t, y, \xi) \quad \text { and } \quad \beta(t)=\Psi(t, y, \xi)
$$

such that $\dot{\alpha}=g_{j}\left(t, y+c_{j} t, \alpha\right)$ and similarly for $\beta$. Using (7.4) we find that

$$
|\alpha(t)-\beta(t)| \leqslant \int_{0}^{t}\left[L_{j, j}|\alpha(s)-\beta(s)|+D_{j}\left(s, y+c_{j} s\right)\right] \mathrm{d} s .
$$

With Gronwall's inequality we find the $\xi$-independent bound

$$
|\alpha(t)-\beta(t)| \leqslant \int_{0}^{t} \mathrm{e}^{L_{j, j}(t-s)} D_{j}\left(s, y+c_{j} s\right) \mathrm{d} s .
$$

Inserting this into (7.5) for $y=x-c_{j}$, we obtain the desired inequality (7.3).

Note that the integral inequalities give rise to an upper bound for $a(t, x)$ in the form $a_{j}(t, x) \leqslant b_{j}(t, x)$ for $t \geqslant 0$ where $b(t, x)$ is the solution of the linear hyperbolic system

$$
\partial_{t} b_{j}+c_{j} \partial_{x} b_{j}=\sum_{k=1}^{d} L_{j, k} b_{k}, \quad \text { for } t \geqslant 0 \text { and } b(0, x)=a(0, x) .
$$

This follows simply by comparing the Picard iterations for $b$ with the corresponding iterations of inequality (7.3). The classical $L^{p}$ estimates for this linear equation lead to the following useful continuity result for $\mathcal{S}_{t}$. 
COROLlaRY 7.3. Let $L=\max \left\{L_{j, k}: j, k=1, \ldots, d\right\}$ and $p \in[1, \infty]$. Then, for each $\bar{\nu}, \bar{\kappa} \in\left(\mathrm{YM}_{\mathrm{c}}(\mathbb{R}, \mathbb{R})\right)^{d}$, we have

$$
\operatorname{dist}_{p}\left(\mathcal{S}_{t}(\bar{\nu}), \mathcal{S}_{t}(\bar{\kappa})\right) \leqslant \mathrm{e}^{L d|t|} \operatorname{dist}_{p}(\bar{\nu}, \bar{\kappa}), \quad t \in \mathbb{R}
$$

where

$$
\operatorname{dist}_{p}(\bar{\nu}, \bar{\kappa})^{p}=\int_{x \in \mathbb{R}} \sum_{j=1}^{d}\left(\mathrm{~d}_{\mathrm{W}}\left(\nu_{j}(x), \kappa_{j}(x)\right)\right)^{p} \mathrm{~d} x,
$$

with the usual modification in the case $p=\infty$.

Of course these results can be localized to finite domains by using the finite propagation speed.

\section{Product measure solutions}

The main goal of this section is the following result.

THEOREM 8.1. Let $f: \mathbb{R}^{d} \rightarrow \mathbb{R}^{d}$ be bounded and globally Lipschitz continuous and $c_{j} \neq c_{k}$ for $j \neq k$. Then, for all $\bar{\nu} \in\left(\mathrm{YM}_{\mathrm{c}}(\mathbb{R}, \mathbb{R})\right)^{d}$, equation (1.1) has a $Y M$ solution $\mu$ in product form, $\mu=\otimes_{j=1}^{d} \mu_{j}$. It is uniquely determined by $\bar{\mu}(t)=\mathcal{S}_{t}(\bar{\nu})$.

This theorem confirms that product-measure solutions play a distinguished role in the set of all YM solutions. However, for $d \geqslant 2$ there are always other YM solutions, even in the linear case, see example 6.2 .

The proof of this theorem is the content of this section and consists of the following steps. First we consider $\nu_{j}$, which can be approximated by functions $v_{j}^{n}(x)=V_{j}\left(x, \kappa_{n}^{(j)} x\right)$ with $\kappa_{n}^{(j)} \rightarrow \infty$ and $V_{j} \in C(\mathbb{R} \times \mathbb{T}, \mathbb{R})$, where $\mathbb{T}=\mathbb{R} / \mathbb{Z}$. This defines solutions $u^{n}$ and we have to control their oscillations in order to exclude resonances. One possible way is to use oscillation frequencies $\kappa_{n}^{(j)}$ of different order, for instance equal to $n^{j}$. We use another approach with all oscillations of the same length-scale by letting $\kappa_{n}^{(j)}=n \omega_{j}$, where the frequency vector $\omega=\left(\omega_{1}, \ldots, \omega_{d}\right)$ satisfies certain non-resonance conditions which are well known in the theory of quasiperiodic motions (cf. [16]). Thus, it is possible to show that $u_{j}^{n}(t, x)$ has asymptotically the form

$$
\tilde{U}_{j}\left(t, x-c_{j} t, n \omega_{j}\left(x-c_{j} t\right)\right)
$$

where $\tilde{U} \in C\left(\mathbb{R}^{2} \times \mathbb{T}, \mathbb{R}^{d}\right)$ is determined by $\left(V_{1}, \ldots, V_{d}\right)$. From this we conclude $u^{n} \stackrel{\mathrm{YM}}{\longrightarrow} \otimes_{1}^{d} \mu_{j}$ with

$$
\left\langle\mu_{j}(t, x), \psi\right\rangle=\int_{z \in \mathbb{T}} \psi\left(\tilde{U}_{j}\left(t, x-c_{j} t, z\right)\right) \mathrm{d} z .
$$

In a final step we use proposition 2.4 to show that all initial data $\bar{\nu}$ can be approximated in the Wasserstein metric dist ${ }_{1}$ by initial data of the above form. With the continuity of $\mathcal{S}_{t}$ in the metric dist 1 we conclude the existence of the YM solution in product form, since convergence in the Wasserstein distance is fine enough to preserve the product structure.

For the frequencies $\omega_{j}$ in $v_{j}^{n}(x)=V_{j}\left(x, n \omega_{j} x\right), V_{j} \in C(\mathbb{R} \times \mathbb{T}, \mathbb{R})$, we use the following non-resonance condition. 
LemmA 8.2. For each $\varepsilon>0$ there exists $\omega \in \mathbb{R}^{d}$ such that for all $j=1, \ldots, d$ and all $p \in \mathbb{Z}^{d} \backslash\{0\}$ the following estimate holds:

$$
\sum_{k=1}^{d} p_{k} \omega_{k} \geqslant \varepsilon|p|^{-d-1}, \quad \text { and if } p_{j}=0 \text { then } \sum_{k \neq j} p_{k} \omega_{k}\left(c_{k}-c_{j}\right) \geqslant \varepsilon|p|^{-d-1} \text {. }
$$

Proof. The result is a little different from the standard small denominator estimates, since we have to satisfy $d+1$ different estimates simultaneously with only one set of $\omega_{j}$. However, the proof is almost identical to the classical one (cf. [16]).

To construct a suitable $\omega$, we study for each $j \in\{0, \ldots, d\}$ and $p$ the size of the set $\Omega(j, p)$ of points $\omega$ that violate (8.1) (here $j=0$ stands for the first estimate not involving $c_{j}$ ). Since $\Omega(j, p)$ lies between two hyperplanes with distance $2 \varepsilon|p|^{-d+1}$, the volume of the intersection of $\Omega(j, p)$ with $B(r)=\{\omega:|\omega| \leqslant r\}$ is less than $2 b_{d-1} \varepsilon r^{d-1}|p|^{-d-1}$, where $b_{d-1}$ is the volume of the unit ball in $\mathbb{R}^{d-1}$. Summing over all $j$ and $p \neq 0$ we find that the volume of $\bigcup_{j, p} \Omega(j, p)$ intersected with $B(r)$ is less than $\hat{b}_{d} r^{d-1} \varepsilon$, where

$$
\hat{b}_{d}=(d+1) b_{d-1} \sum_{p \neq 0}|p|^{-d-1}<\infty .
$$

Hence, making $r$ sufficiently large there is an $\omega \in B(r)$ that does not lie in any of the sets $\Omega(j, p)$.

We now study weak solutions $u^{n}$ of (1.1) that satisfy the initial conditions

$$
u_{j}^{n}(0, x)=V_{j}\left(x, n \omega_{j} x\right), \quad j=1, \ldots, d .
$$

With inspiration from $[10,13]$ we construct an auxiliary solution $\tilde{u}^{n}$ in the form $\tilde{u}_{j}^{n}(t, x)=\tilde{U}_{j}\left(t, x-c_{j} t, n \omega_{j}\left(x-c_{j} t\right)\right)$, where the functions $\tilde{U}_{j}$ satisfy

$$
\begin{aligned}
& \tilde{U}_{j}\left(t, y_{j}, z_{j}\right)=V_{j}\left(y_{j}, z_{j}\right) \\
& \quad+\int_{s=0}^{t} f_{j}^{*}\left(\tilde{U}_{1}\left(s, y_{j, 1}(s), \cdot\right), \ldots, \tilde{U}_{j}\left(s, y_{1}, z_{j}\right), \tilde{U}_{j+1}\left(s, y_{j, j+1}(s), \cdot\right), \ldots\right) \mathrm{d} s
\end{aligned}
$$

where

$$
y_{j, k}(s)=y_{j}-\left(c_{k}-c_{j}\right) s, \quad z_{\nexists j}=\left(z_{1}, \ldots, z_{j-1}, z_{j+1}, \ldots\right) \in \mathbb{T}^{d-1},
$$

and $f_{j}^{*}$ is defined as

$$
\begin{aligned}
f_{j}^{*}\left(\left(\tilde{U}_{1}(\cdot), \ldots, \tilde{U}_{j-1}(\cdot), u_{j}, \tilde{U}_{j+1}(\cdot), \ldots, \tilde{U}_{d}(\cdot)\right)\right) \\
\quad=\int_{z_{\nexists j} \in \mathbb{T}^{d-1}} f\left(\tilde{U}_{1}\left(z_{1}\right), \ldots, \tilde{U}_{j-1}\left(z_{j-1}\right), u_{j}, \tilde{U}_{j+1}\left(z_{j+1}\right), \ldots, \tilde{U}_{d}\left(z_{d}\right)\right) \mathrm{d} z_{\not \jmath j} .
\end{aligned}
$$

Proposition 8.3. Assume $V_{j} \in C(\mathbb{R} \times \mathbb{T}, \mathbb{R})$ and $f: \mathbb{R}^{d} \rightarrow \mathbb{R}^{d}$ is globally Lipschitz continuous. Then, (8.3) has a unique global solution $\tilde{U} \in C\left(\mathbb{R}^{2} \times \mathbb{T}, \mathbb{R}^{d}\right)$.

Proof. This result follows exactly by the scheme of the proof for theorem 4.1. 
From the construction of $\tilde{U}$ and $\tilde{u}^{n}$ it is clear that the limits $\tilde{u}_{j}^{n} \stackrel{\mathrm{YM}}{\longrightarrow} \mu_{j}$ exist with

$$
\left\langle\mu_{j}(t, x, \mathrm{~d} v), \psi(v)\right\rangle_{\mathbb{R}}=\int_{z_{j}=0}^{1} \psi\left(\tilde{U}_{j}\left(t, x, z_{j}\right)\right) \mathrm{d} z_{j},
$$

and that the marginals, $\bar{\mu}$, satisfy the non-resonance system $(3.11)$, that is $\bar{\mu}(t)=$ $\mathcal{S}_{t}(\bar{\nu})$. However, the following lemma tells us more: the YM associated to $\tilde{u}^{n}$ is in fact the product measure generated by the marginals.

Proposition 8.4. Let $\omega \in \mathbb{R}^{d}$ satisfy (8.1), assume $\tilde{U} \in C\left(\mathbb{R}^{2} \times \mathbb{T}, \mathbb{R}^{d}\right)$, and define $\tilde{u}^{n}$ via

$$
\tilde{u}_{j}^{n}(t, x)=\tilde{U}\left(t, x-c_{j} t, n \omega_{j}\left(x-c_{j} t\right)\right)
$$

Then, $\tilde{u}^{n} \stackrel{Y M}{\longrightarrow} \mu=\otimes_{j=1}^{d} \mu_{j}$ with $\mu_{j}$ given in (8.4).

Proof. For each $\phi$ and $\psi_{j}$ we have to show that

$$
\int_{\mathbb{R}^{2}} \phi(t, x) \prod_{j} \psi_{j}\left(\tilde{u}_{j}^{n}(t, x)\right) \mathrm{d}(t, x)
$$

converges towards

$$
\int_{\mathbb{R}^{2}} \phi(t, x) \prod_{j} \int_{z_{j} \in \mathbb{T}} \psi_{j}\left(\tilde{U}\left(t, x-c_{j} t, z_{j}\right)\right) \mathrm{d} z_{j} \mathrm{~d}(t, x) .
$$

However, this follows immediately from the special form of $\tilde{u}^{n}$ and theorem B.1 below, if we set

$$
g(t, x, z)=\prod_{j=1}^{d} \psi_{j}\left(\tilde{U}_{j}\left(t, x-c_{j} t, z_{j}\right)\right)
$$

It remains to compare the true solutions $u^{n}$ of (1.1) with initial conditions (8.2) with our auxiliary functions $\tilde{u}^{n}$. If we show $u^{n}-\tilde{u}^{n} \rightarrow 0$ in $L_{\text {loc }}^{\infty}\left(\mathbb{R}^{2}\right)$, then $u^{n}$ converges to the same YM as $\tilde{u}^{n}$, which is the product measure $\otimes \mu_{j}$. Since, on the one hand, each $u^{n}$ is a classical solution and, on the other hand, $\bar{\mu}$ satisfies the non-resonance system (3.11), we can conclude that (1.1) always has one YM solution that is a product measure and, thus, the unique solution of (3.11).

Proposition 8.5. Let $f$ be Lipschitz continuous in $u$ and let $\omega \in \mathbb{R}^{d}$ satisfy (8.1). Assume that the initial condition $\bar{\nu} \in\left(\mathrm{YM}_{\mathrm{c}}(\mathbb{R}, \mathbb{R})\right)^{d}$ is given via $V \in C(\mathbb{R} \times$ $\left.\mathbb{T}, \mathbb{R}^{d}\right)$, such that $v_{j}^{n}(x)=V_{j}\left(x, n \omega_{j} x\right) \stackrel{Y M}{\longrightarrow} \nu_{j}$. Then, the solutions $u^{n}$ of (1.1) with $u^{n}(0, x)=v^{n}(x)$ satisfy $u^{n} \stackrel{Y M}{\longrightarrow} \mu$ and $\mu$ has the form $\mu=\otimes \mu_{j}$ with $\bar{\mu}(t)=\mathcal{S}_{t}(\bar{\nu})$.

Proof. It remains to show that the difference $w^{n}=u^{n}-\tilde{u}^{n}$ converges strongly to zero. This will be done by deriving a Gronwall estimate for $w^{n}$. The integral equation for $u_{j}^{n}$, in the case $j=1$, reads

$$
u_{1}^{n}\left(t, y_{1}\right)=V\left(y_{1}, n \omega_{1} y_{1}\right)+\int_{0}^{t} f_{1}\left(u_{1}^{n}\left(s, y_{1}\right), u_{2}^{n}\left(s, y_{1,2}(s)\right), \ldots\right) \mathrm{d} s
$$


with $y_{j}=x-c_{j} t$ and $y_{j, k}(s)=y_{j}-\left(c_{k}-c_{j}\right) s$. We define a sequence of auxiliary functions $U^{n} \in C\left(\mathbb{R}^{2} \times \mathbb{T}, \mathbb{R}^{d}\right)$ satisfying

$$
U_{1}^{n}\left(t, y_{1}, z_{1}\right)=V\left(y_{1}, z_{1}\right)+\int_{0}^{t} f_{1}\left(U_{1}^{n}\left(s, y_{1}, z_{1}\right), U_{2}^{n}\left(s, y_{1,2}(s), n \omega_{2} y_{1,2}(s)\right), \ldots\right) \mathrm{d} s,
$$

and similarly for $U_{j}^{n}$. The existence of these functions $U^{n}$, follows again by a contraction mapping argument as in the proof of theorem 4.1. The construction is done exactly such that

$u_{j}^{n}(t, x)=U^{n}\left(t, x-c_{j} t, n \omega_{j}\left(x-c_{j} t\right)\right)$ and $w_{j}^{n}(t, x)=W^{n}\left(t, x-c_{j} t, n \omega_{j}\left(x-c_{j} t\right)\right)$, where $W^{n}=U^{n}-\tilde{U}$. Taking the difference of the two integral equations, the difference $W^{n}$ is estimated by Lipschitz continuity as follows:

$$
\left|W_{j}^{n}\left(t, y_{j}, z_{j}\right)\right| \leqslant \int_{s=0}^{t} L \sum_{k=1}^{d} \sup _{z_{k} \in \mathbb{T}}\left\{\left|W_{k}^{n}\left(s, y_{j, k}(s), z_{k}\right)\right|\right\} \mathrm{d} s+\left|\int_{0}^{t} \rho_{j}\left(n, s, y_{j}, z_{j}\right) \mathrm{d} s\right|,
$$

where $\rho_{1}\left(n, s, y_{1}, z_{1}\right)$ is defined as

$$
\begin{aligned}
f_{1}\left(\tilde{U}_{1}\left(s, y_{1}, z_{1}\right), \tilde{U}_{2}\left(s, y_{1,2}(s)\right.\right. & \left.\left.n \omega_{2} y_{1,2}(s)\right), \ldots\right) \\
& -\int_{z_{\not 1} \in \mathbb{T}^{d-1}} f_{1}\left(\tilde{U}_{1}\left(s, y_{1}, z_{1}\right), \tilde{U}_{2}\left(s, y_{1,2}(s), z_{2}\right), \ldots\right) \mathrm{d} z_{\not \supset 1},
\end{aligned}
$$

and similarly for $\rho_{j}\left(n, s, y_{j}\right)$. With $c_{\Delta}=\max \left\{\left|c_{j}-c_{k}\right|: j, k=1, \ldots, d\right\}$ and any given $T>0$ we thus have

$$
\alpha^{n}(t) \leqslant L \int_{0}^{t} \alpha^{n}(s) \mathrm{d} s+R(n, t)
$$

for $t \in[0, T]$, where

$$
\alpha^{n}(t)=\left\|W^{n}(t)\right\|_{L^{\infty}\left(\left[c_{\Delta}(t-T), c_{\Delta}(T-t)\right] \times \mathbb{T}\right)}
$$

and

$$
\begin{aligned}
& R(n, t)=\sup \left\{\left|\int_{0}^{\tau} \rho_{j}\left(n, s, y_{j}, z_{j}\right) \mathrm{d} s\right|:\right. \\
& \left.\quad j=1, \ldots, d, z_{j} \in \mathbb{T}, 0 \leqslant \tau \leqslant t,\left|y_{j}\right| \leqslant c_{\Delta}(t-\tau)\right\} .
\end{aligned}
$$

Gronwall's estimate and the monotonicity of $R$ in $t$ yields $\alpha^{n}(t) \leqslant \mathrm{e}^{L t} R(n, t)$.

Hence, it is sufficient to show that $R(n, t)$ converges to 0 for $n \rightarrow \infty$. But this is a consequence of theorem B.2 below, since $\rho_{1}$ has the form

$$
\rho_{1}\left(n, s, y_{1}, z_{1}\right)=g_{1}\left(s, y_{1}, z_{1}, n \omega_{2}\left(y_{1}+\left(c_{2}-c_{1}\right) s\right), \ldots, n \omega_{d}\left(y_{1}+\left(c_{d}-c_{1}\right) s\right)\right),
$$

where $g_{1} \in C\left(\mathbb{R}^{2} \times \mathbb{T}^{d}\right)$ with

$$
\int_{z_{\not 1} \in \mathbb{T}^{d-1}} g\left(s, y_{1}, z\right) \mathrm{d} z_{\not \ngtr 1}=0,
$$

by the definition of $\rho_{1}$. Note that $g$ only needs to be considered on the compact domain

$$
\left\{\left(s, y_{1}, z\right): s \in[0, T],\left|y_{1}\right| \leqslant c_{\Delta}(t-s), z \in \mathbb{T}^{d}\right\},
$$

where it is uniformly continuous. 
We are now ready to prove the main result by approximation in the Wasserstein metric.

Proof of theorem 8.1. For $\bar{\nu} \in\left(\mathrm{YM}_{\mathrm{C}}(\mathbb{R}, \mathbb{R})\right)^{d}$ there exists, according to proposition 2.4, a sequence $\bar{\gamma}^{n}$ such that

$$
\operatorname{dist}_{L^{1}((-n, n))}\left(\bar{\gamma}^{n}, \bar{\nu}\right)<1 / n
$$

and

$$
\left\langle\gamma_{j}^{n}(x), \psi\right\rangle=\int_{z \in \mathbb{T}} \psi\left(V_{j}^{n}(x, z)\right) \mathrm{d} z,
$$

with $V_{j}^{n} \in C(\mathbb{R} \times \mathbb{T}, \mathbb{R})$. (Here we used the subscript $L^{1}((a, b))$ in the Wasserstein distance to indicate that this approximation is only valid on a bounded domain.) Thus, proposition 8.5 is applicable, which provides YM solutions $\mu^{n}=\otimes_{j=1}^{d} \mu_{j}^{n}$ with $\mu^{n}(t)=\mathcal{S}_{t}\left(\bar{\gamma}^{n}\right)$. The continuity of $\mathcal{S}_{t}$, shown in corollary 7.3 , gives

$$
\operatorname{dist}_{L^{1}((a, b))}\left(\bar{\mu}^{n}(t), \mu^{*}(t)\right) \rightarrow 0, \quad \text { with } \mu^{*}(t)=\mathcal{S}_{t}(\bar{\nu}),
$$

for all bounded intervals $(a, b) \subset \mathbb{R}$. With lemma 2.1 this implies

$$
\mathrm{d}_{\mathrm{W}}\left(\mu^{n}(t, x), \otimes \mu_{j}^{*}(t, x)\right)=\mathrm{d}_{\mathrm{W}}\left(\otimes \mu_{j}^{n}(t, x), \otimes \mu_{j}^{*}(t, x)\right)=\sum_{k=1}^{d} \mathrm{~d}_{\mathrm{W}}\left(\mu_{k}^{n}(t, x), \mu_{k}^{*}\right) \rightarrow 0,
$$

for a.e. $(t, x)$. Hence, $\mu^{n} \stackrel{*}{\rightarrow} \mu^{*}=\otimes \mu_{j}^{*}$ and the weak* closedness of the set of YM solutions tells us that $\mu^{*}$ is the desired solution.

\section{Acknowledgments}

Research partly supported by VW-Stiftung under grant I/70284 (Spannungs-und verzerrungsbedingte Phasenübergänge in Ingenieurswerkstoffen) and by the MaxPlanck-Institut für Mathematik in den Naturwissenschaften, Leipzig.

The author is grateful to J. Batt, F. Otto, G. Rein and F. Theil for stimulating discussions. He is greatly indebted to L. Tartar for providing further insights and motivations via [24].

\section{Appendix A. $H$-measures and multilinear products}

In this appendix we provide a result concerning the product of three sequences of weakly converging functions. To this end we use the $H$-measure as introduced in [22], the same object is called defect measure in [8]. Let $u^{n}$ be a sequence converging weakly to zero in $L^{2}\left(\mathbb{R}^{m}, \mathbb{R}^{d}\right)$, then there exists a subsequence $n_{k}$ and a family $H_{i j}$ of complex-valued Radon measures on $\mathbb{R}^{m} \times \mathbb{S}^{m-1}$ such that for every test function $\phi_{1}, \phi_{2} \in C_{0}\left(\mathbb{R}^{m}, \mathbb{C}\right)$ and every function $\Phi \in C\left(\mathbb{S}^{m-1}, \mathbb{C}\right)$ the identity

$$
\begin{aligned}
& \left\langle H_{i j}(\mathrm{~d} x, \mathrm{~d} \xi), \phi_{1}(x) \overline{\phi_{2}(x)} \Phi(\omega)\right\rangle_{(x, \omega) \in \mathbb{R}^{m} \times \mathbb{S}^{m-1}} \\
& \quad=\lim _{k \rightarrow \infty} \int_{\xi \in \mathbb{R}^{m}}\left[\mathcal{F}_{m}\left(\phi_{1} u_{i}^{n_{k}}\right)(\xi)\right] \overline{\left[\mathcal{F}\left(\phi_{2} u_{j}^{n_{k}}\right)(\xi)\right]} \Phi(\xi /|\xi|) \mathrm{d} \xi,
\end{aligned}
$$

holds, where the overline denotes complex conjugation and $\mathcal{F}_{m}$ the $m$-dimensional Fourier transform. The matrix $\left(H_{i j}\right)$ of complex-valued measures is called the $H$ measure of the subsequence $u^{n_{k}}$. The function $\Phi \in C\left(\mathbb{S}^{n-1}\right)$ allows us to detect in which direction, $\xi /|\xi|$, the oscillations are dominant. 
Our application involves in fact sequences that are bounded in $L_{\text {loc }}^{\infty}\left(\mathbb{R}^{m}\right)$. Therefore no concentration effects can occur, and we consider $H_{i j}$ as a family $\mu_{i j}(x) \in$ $\mathrm{M}\left(\mathbb{S}^{m-1}\right)$ such that

$$
\left\langle H_{i j}(\mathrm{~d} x, \mathrm{~d} \omega), \phi(x) \Phi(\omega)\right\rangle=\int_{\mathbb{R}^{m}} \phi(x)\left\langle\mu_{i j}(x, \mathrm{~d} \omega), \Phi(\omega)\right\rangle \mathrm{d} x .
$$

In particular,

$$
u_{i}^{n_{k}} u_{j}^{n_{k}} \stackrel{*}{\rightarrow}\left\langle\mu_{i j}(\cdot), 1_{\mathbb{S}^{m-1}}\right\rangle,
$$

which follows easily from the definition if $\Phi \equiv 1$, since then the Fourier transform can be undone by Parseval's identity. We also have positive definiteness, $\bar{\mu}_{i j}=\mu_{j i}$ and

$$
\sum_{i, j=1}^{d} \phi_{i} \bar{\phi}_{j} \mu_{i j} \geqslant 0
$$

for all $\phi_{k}$, which we will use in the form

$$
\left|\left\langle\mu_{i j}, \Phi\right\rangle\right|^{2} \leqslant\left\langle\mu_{i i}, \Phi\right\rangle\left\langle\mu_{j j}, \Phi\right\rangle,
$$

for all $\Phi \in C\left(\mathbb{S}^{m-1}\right)$. This and all the following estimates have to be understood as estimates for scalar measurable functions which hold for a.e. $x \in \mathbb{R}^{m}$.

For Young measure solutions of semilinear hyperbolic systems we gain additional information by using the differential equations. Now we restrict the analysis to $x=\left(x_{1}, x_{2}\right) \in \mathbb{R}^{2}$.

Lemma A.1. Let $\theta=(c, s) \in \mathbb{S}^{1}$. Assume that $u_{j}^{n}$ as well as $\left(s \partial_{x_{1}}-c \partial_{x_{2}}\right) u_{j}^{n}=g^{n}$ are bounded sequences in $L_{\mathrm{loc}}^{\infty}\left(\mathbb{R}^{2}, \mathbb{R}\right)$ converging weakly to zero and that $\left(u_{j}^{n}\right)^{2} \stackrel{*}{\rightarrow} v_{j}$. Then, the associated $H$-measure is

$$
\mu_{j j}(x)=v_{j}(x) \frac{1}{2}\left(\delta_{\theta}+\delta_{-\theta}\right) .
$$

This results says that the oscillations of $u_{j}^{n}$ are perpendicular to the characteristic direction and the amplitude is given by the weak limit $v_{j}$ of $\left(u_{j}^{n}\right)^{2}$. The fact that $\mu_{j j}$ is localized in the points $\theta$ and $-\theta$ is the contents of [22, theorem 1.6]. The mass is equally distributed between the two points since $\mu_{j j}$ is invariant under the involution $-\mathrm{id}_{\mathbb{S}^{1}}$.

The classical result of compactness through compensation follows easily from (A 2) and lemma A.1 if $\theta_{i} \neq \pm \theta_{j}$ : from (A 2) we know that the support of $\mu_{i j}(x)$ lies in the intersection of the supports of $\mu_{i i}(x)$ and $\mu_{j j}(x)$, but this is empty and hence $\mu_{i j} \equiv 0$, which means $u_{i}^{n} u_{j}^{n} \stackrel{*}{\rightarrow} 0$.

Theorem A.2. Let $\theta_{j}=\left(c_{j}, s_{j}\right) \in \mathbb{S}^{1}$ for $j=1,2,3$ such that $\theta_{j} \neq \pm \theta_{i}$ for $i \neq j$. Assume that $\left(u_{1}^{n}, u_{2}^{n}, u_{3}^{n}\right)$ as well as $\left(s_{j} \partial_{x_{1}}-c_{j} \partial_{x_{2}}\right) u_{j}^{n}=g_{j}^{n}$ be bounded sequences in $L_{\mathrm{loc}}^{\infty}\left(\mathbb{R}^{2}\right)$ converging weakly to zero. Then,

$$
\left[\left(u_{j}^{n}\right)^{2} \stackrel{*}{\rightarrow} v_{j} \text { and } u_{1}^{n} u_{2}^{n} u_{3}^{n} \stackrel{*}{\rightarrow} \gamma\right] \Longrightarrow\left[\gamma^{2} \leqslant \frac{1}{2} v_{1} v_{2} v_{3}\right] .
$$

Proof. Since $H$-measures are constructed to deal with bilinear products we define $u_{4}^{n}=u_{1}^{n} u_{2}^{n}$. Using $\theta_{1} \neq \pm \theta_{2}$ the compactness through compensation (cf. theorem 5.1) gives

$$
u_{4}^{n}=u_{1}^{n} u_{2}^{n} \stackrel{*}{\rightarrow} 0, \quad\left(u_{4}^{n}\right)^{2}=\left(u_{1}^{n}\right)^{2}\left(u_{2}^{n}\right)^{2} \stackrel{*}{\rightarrow} v_{1} v_{2} .
$$


We now apply the $H$-measure theory to the pair $\left(u_{3}^{n}, u_{4}^{n}\right)$ and obtain, after choosing a suitable subsequence, the measures $\mu_{33}, \mu_{34}$ and $\mu_{44}$ such that

$$
\gamma=\left\langle\mu_{34}, 1_{\mathbb{S}^{1}}\right\rangle, \quad v_{3}=\left\langle\mu_{33}, 1_{\mathbb{S}^{1}}\right\rangle, \quad v_{1} v_{2}=\left\langle\mu_{44}, 1_{\mathbb{S}^{1}}\right\rangle .
$$

Together with (A 2) this implies the desired result except for the factor $\frac{1}{2}$.

To obtain the better result we use that $\mu_{33}$ is concentrated with equal weight in $\theta_{3}$ and $-\theta_{3}$. Again using (A 2) we conclude that $\mu_{34}$ is concentrated in these two points and thus,

$$
\gamma^{2}=\left\langle\mu_{34}, 1_{\mathbb{S}_{1}}\right\rangle^{2}=\mu_{34}\left(\left\{\theta_{3},-\theta_{3}\right\}\right)^{2} \leqslant \mu_{33}\left(\left\{\theta_{3},-\theta_{3}\right\}\right) \mu_{44}\left(\left\{\theta_{3},-\theta_{3}\right\}\right) .
$$

Since $v_{3}=\mu_{33}\left(\left\{\theta_{3},-\theta_{3}\right\}\right)$ the result is established if we show that $\mu_{44}\left(\left\{\theta_{3},-\theta_{3}\right\}\right) \leqslant$ $\frac{1}{2} v_{1} v_{2}$. This will follow from an additional symmetry of $\mu_{44}$ that stems from the fact that $u_{4}^{n}$ is the product of two functions that have oscillations in linearly independent directions. Define the map $T: \mathbb{R}^{2} \rightarrow \mathbb{R}^{2}$ via

$T \xi=\alpha\left(R \theta_{2} \cdot \xi\right) \theta_{1}+\alpha\left(R \theta_{1} \cdot \xi\right) \theta_{2}, \quad$ with $R=\left(\begin{array}{cc}0 & -1 \\ 1 & 0\end{array}\right) \quad$ and $\quad \alpha=1 /\left(c_{1} s_{2}-s_{1} c_{2}\right)$, and the induced mapping

$$
\mathcal{T}: \mathbb{S}^{1} \rightarrow \mathbb{S}^{1} ; \omega \mapsto \mathcal{T}(\omega)=T \omega /|T \omega|
$$

If the symmetry

$$
\mathcal{T}_{*} \mu_{44}(x)=\mu_{44}(x), \quad \text { for a.e. } x \in \mathbb{R}^{2}
$$

holds, we have $\mu_{44}\left(\left\{\mathcal{T}\left( \pm \theta_{3}\right)\right\}\right)=\mu_{44}\left(\left\{\theta_{3}\right\}\right)$. However, since $\theta_{3}$ is different from $\pm \theta_{1}, \pm \theta_{2}$ we have four points of equal mass. Hence,

$$
\mu_{44}\left(\left\{\theta_{3},-\theta_{3}\right\}\right)=\frac{1}{2} \mu_{44}\left(\left\{\theta_{3},-\theta_{3}, \mathcal{T}\left(\theta_{3}\right),-\mathcal{T}\left(\theta_{3}\right)\right\}\right) \leqslant \frac{1}{2}\left\langle\mu_{44}, 1_{\mathbb{S} 1}\right\rangle=\frac{1}{2} v_{1} v_{2} .
$$

It suffices to prove (A 4 ) for Lebesgue points, since they form a set of full measure. A point $y$ is called a Lebesgue point if

$$
\lim _{\varepsilon \rightarrow 0} \frac{1}{\pi \varepsilon^{2}} \int_{|x-y|<\varepsilon}\left\langle\mu_{44}(x), \Phi\right\rangle \mathrm{d} x=\left\langle\mu_{44}(y), \Phi\right\rangle,
$$

for each $\Phi \in C\left(\mathbb{S}^{1}\right)$. Our next construction reduces the analysis to the case $g_{j}^{n} \equiv 0$ which makes the functions constant along characteristics.

Take any such $y$ and define the functions

$$
\tilde{u}_{j}^{n}(x)=u_{j}^{n}\left(y+\theta_{j} \cdot(x-y) \theta_{j}\right),
$$

which satisfy $\left|u^{n}(x)-\tilde{u}^{n}(x)\right|=\mathcal{O}(|x-y|)$ uniformly in $n$ by our assumptions. To see this, use the differential equation along the characteristics and integrate $g_{j}^{n}$. Extracting a suitable subsequence we find $\tilde{u}_{j}^{n} \stackrel{*}{\rightarrow} e_{j}$ with $\left|e_{j}(x)\right|=\mathcal{O}(|x-y|)$.

Consider $\hat{u}_{j}^{n}(x)=\tilde{u}_{j}^{n}(x)-e_{j}(x)$, then

$$
\left(s_{j} \partial_{x_{1}}-c_{j} \partial_{x_{2}}\right) \hat{u}_{i}^{n}=0, \quad \hat{u}_{j}^{n} \stackrel{*}{\rightarrow} 0, \quad\left|u_{j}^{n}(x)-\hat{u}_{j}^{n}(x)\right|=\mathcal{O}(|x-y|) .
$$

Extracting a further subsequence $\hat{u}_{4}^{n}=\hat{u}_{1}^{n} \hat{u}_{2}^{n}$ defines an $H$-measure $\hat{\mu}_{44}$ that satisfies

$$
\int_{|x-y| \leqslant \varepsilon}\left\langle\hat{\mu}_{44}(x), \Phi\right\rangle \mathrm{d} x=\int_{|x-y| \leqslant \varepsilon}\left\langle\mu_{44}(x), \Phi\right\rangle \mathrm{d} x+\mathcal{O}\left(\varepsilon^{3}\right),
$$


by comparing the arguments in the respective limits in (A 1). We find that $y$ is also a Lebesgue point for $\hat{\mu}_{44}$ and $\hat{\mu}_{44}(y)=\mu_{44}(y)$. Hence, it suffices to show (A 4) for $\hat{\mu}_{44}(y)$. Without loss of generality we can also take $y=0$.

The symmetry of $\hat{\mu}_{44}$ deduces from the product form

$$
\hat{u}_{4}^{n}(x)=w_{1}^{n}\left(\theta_{1} \cdot x\right) w_{2}^{n}\left(\theta_{2} \cdot x\right),
$$

where $w_{j}^{n}(s)=u_{j}^{n}\left(s \theta_{j}\right)$. For test functions

$$
\phi(x)=\psi_{1}\left(\theta_{1} \cdot x\right) \psi_{2}\left(\theta_{2} \cdot x\right)
$$

with $\psi_{j} \in C_{0}(\mathbb{R}, \mathbb{C})$ we find

$$
\mathcal{F}_{2}\left(\phi \hat{u}_{4}^{n}\right)(\xi)=\operatorname{det}(A)\left[\mathcal{F}_{1}\left(\psi_{1} w_{1}^{n}\right)\left(A e_{1} \cdot \xi\right)\right]\left[\mathcal{F}_{1}\left(\psi_{2} w_{2}^{n}\right)\left(A e_{2} \cdot \xi\right)\right],
$$

where $A$ is the inverse of the matrix $\left(\theta_{1}, \theta_{2}\right)^{\mathrm{T}}$. In particular, $A e_{j}=(-1)^{j} \alpha R \theta_{j}$ and $\operatorname{det} A=\alpha$. Using the trivial relation

$$
\mathcal{F}_{m}(z)(-\eta)=\overline{\mathcal{F}_{m}(\bar{z})(\eta)}
$$

we can now change the sign of $A e_{j} \cdot \xi$ for $j=1$ and 2 independently. With $T^{\mathrm{T}} A e_{j}=$ $(-1)^{j} A e_{j}$ we conclude that

$$
\begin{aligned}
\int_{\mathbb{R}^{2}}\left|\mathcal{F}_{2}\left(\phi \hat{u}_{4}^{n}\right)(\xi)\right|^{2} \Phi(\mathcal{T}(\xi /|\xi|)) \mathrm{d} \xi & =\int_{\mathbb{R}^{2}}\left|\mathcal{F}_{2}\left(\phi \hat{u}_{4}^{n}\right)(T \xi)\right|^{2} \Phi(\mathcal{T}(\xi /|\xi|)) \mathrm{d} \xi \\
& \left.=\int_{\mathbb{R}^{2}}\left|\mathcal{F}_{2}\left(\phi \hat{u}_{4}^{n}\right)(\eta)\right|^{2} \Phi(\eta /|\eta|)\right) \mathrm{d} \eta
\end{aligned}
$$

where the substitution $\eta=T \xi$ was used. Taking the limit $n \rightarrow \infty$ we obtain the desired result since it suffices to test with functions in product form.

We note that the above constant $\frac{1}{2}$ in (A 3 ) is optimal. It is attained for the functions $u_{j}^{n}(t, x)=\cos \left(b_{j} n\left(x-c_{j} t\right)\right)$ with $b=(1,2,1)$ and $c=(1,0,-1)$, where $v_{j} \equiv \frac{1}{2}$ and $\gamma=\frac{1}{4}$. For products of four functions we have the following result.

THEOREM A.3. Let $\theta_{j}=\left(c_{j}, s_{j}\right) \in \mathbb{S}^{1}$ for $j=1, \ldots, 4$ such that $\theta_{1} \neq \pm \theta_{2}$ and $\theta_{3} \neq \pm \theta_{4}$. Assume that $u_{j}^{n}$ as well as $\left(s_{j} \partial_{x_{1}}-c_{j} \partial_{x_{2}}\right) u_{j}^{n}=g_{j}^{n}$ be bounded sequences in $L_{\mathrm{loc}}^{\infty}\left(\mathbb{R}^{2}\right)$ converging weakly to zero. Then,

$$
\left[\left(u_{j}^{n}\right)^{2} \stackrel{*}{\rightarrow} v_{j} \text { and } u_{1}^{n} u_{2}^{n} u_{3}^{n} u_{4}^{n} \stackrel{*}{\rightarrow} \gamma\right] \Longrightarrow\left[\gamma^{2} \leqslant v_{1} v_{2} v_{3} v_{4}\right] .
$$

Proof. As in the proof of theorem A.2 we can associate $H$-measures $\mu_{k l}, k, l=5,6$, to the sequences $u_{5}^{n}=u_{1}^{n} u_{2}^{n}$ and $u_{6}^{n}=u_{3}^{n} u_{4}^{n}$ such that

$$
\gamma=\left\langle\mu_{56}, 1_{\mathbb{S}^{1}}\right\rangle, \quad v_{1} v_{2}=\left\langle\mu_{55}, 1_{\mathbb{S}^{1}}\right\rangle, \quad v_{3} v_{4}=\left\langle\mu_{66}, 1_{\mathbb{S}^{1}}\right\rangle .
$$

Now the result is a consequence of (A 2).

\section{Appendix B. Quasiperiodic averaging}

THEOREM B.1. Let $\omega \in \mathbb{R}^{d}$ be chosen such that (8.1) holds. If $g \in C^{0}\left(\mathbb{R}^{2} \times \mathbb{T}^{d}, \mathbb{R}\right)$, then the sequence $h^{n}$, defined by

$$
h^{n}(t, x)=g\left(t, x, n \omega_{1}\left(x-c_{1} t\right), \ldots, n \omega_{d}\left(x-c_{d} t\right)\right),
$$


satisfies

$$
\int_{\mathbb{R}^{2}} \phi(t, x) h^{n}(t, x) \mathrm{d}(t, x) \rightarrow \int_{\mathbb{R}^{2}} \phi(t, x) \int_{\mathbb{T}^{d}} g(t, x, z) \mathrm{d} z \mathrm{~d}(t, x), \quad \text { for all } \phi \in C_{\mathrm{c}}^{0}\left(\mathbb{R}^{2}\right) \text {. }
$$

Proof. We proceed as in classical ergodic theory [16], and first assume that $g$ is $(d+1)$-times continuously differentiable in the $z$ variable. Then, $g$ has a Fourier series expansion

$$
g(t, x, z)=\sum_{p \in \mathbb{Z}^{d}} g_{p}(t, x) \mathrm{e}^{2 \pi \mathrm{i} p \cdot z}
$$

whose coefficients are continuous in $(t, x)$ and satisfy the estimate $\left|g_{p}\right| \leqslant C(1+$ $|p|)^{-d-1}$. Note that the right-hand side in (B 1$)$ is exactly the integral of $\phi g_{0}$. Hence, after subtracting the average we may assume that $g_{0} \equiv 0$ such that it remains to show that the left-hand side converges to zero.

Given a positive $\varepsilon$ there is, by uniform continuity, a positive $\delta$ such that

$$
|\phi(t, x)-\phi(s, y)|,|g(t, x, z)-g(s, y, z)| \leqslant \varepsilon /\left(2\|\phi\|_{\infty}\|g\|_{\infty}\right),
$$

whenever $|t-s|+|x-y| \leqslant \delta$. Assume $\operatorname{sppt}(\phi) \subset[-N \delta, N \delta]^{2}$, then

$$
\begin{aligned}
& \mid \int_{\mathbb{R}^{2}} \phi h^{n} \mathrm{~d}(t, x) \\
& \quad-\sum_{k, l=-N}^{N-1} \int_{Q_{k, l}} \phi(k \delta, l \delta) g\left(k \delta, l \delta, n \omega_{1}\left(x-c_{1} t\right), \ldots, n \omega_{d}\left(x-c_{d} t\right)\right) \mathrm{d}(t, x) \mid \leqslant \frac{1}{2} \varepsilon
\end{aligned}
$$

where $Q_{k, l}=[k \delta,(k+1) \delta] \times[l \delta,(l+1) \delta]$. Thus, it remains to make each of the integrals over $Q_{k, l}$ small. Here, we use $n \rightarrow \infty, g_{0}(k \delta, j \delta)=0$ and the small divisor estimate (8.1). Using that $Q_{k, l}$ is a square and the estimate

$$
\left|\int_{0}^{\delta} \mathrm{e}^{\mathrm{i} a s} \mathrm{~d} s\right| \leqslant \min \{\delta, 1 / a\}
$$

we find that

$$
\begin{aligned}
& \left|\int_{Q_{k, l}} \sum_{p \neq 0} g_{p}(k \delta, l \delta) \mathrm{e}^{2 \pi \mathrm{i} n p \cdot[x \omega-t C \omega]} \mathrm{d}(t, x)\right| \\
& \quad \leqslant \sum_{p \neq 0}\left|g_{p}\right| \min \{\delta, 1 /(2 \pi n|p \cdot \omega|)\} \min \{\delta, 1 /(2 \pi n|p \cdot C \omega|)\} \\
& \quad \leqslant \sum_{p \neq 0} \hat{C}|p|^{-d-1} \min \left\{\delta,|p|^{d+1} / n\right\} \delta \leqslant \tilde{C}(\delta) n^{-1 /(d+1)} .
\end{aligned}
$$

This proves the result since $\delta$ and $N$ are fixed (for fixed $\varepsilon$ ) and, hence, each integral over $Q_{k, l}$ can be made smaller than $\varepsilon /\left(N^{2}\right)$, such that the whole integral is bounded by $\varepsilon$. This proves the assertion for the differentiable case.

We know that the linear mapping $A: g \mapsto\left(\int \phi h^{n} \mathrm{~d}(t, x)\right)_{n \in \mathbb{N}}$ produces, for every continuous $g$, a bounded sequence. Since $g$ is uniformly continuous on the support of $\phi$ it can be approximated by a sequence $g_{(m)}$ of $C^{d+1}$ functions, all having zero 
mean. However, because of the above arguments all the sequences

$$
A g_{(m)}=\left(\int \phi h_{(m)}^{n} \mathrm{~d}(t, x)\right)_{n \in \mathbb{N}},
$$

converge to 0 for $n \rightarrow \infty$. Whence also the sequence $A g$ has to converge to 0 .

TheOREM B.2. Let $g \in C\left(\mathbb{R}^{2} \times \mathbb{T}^{d}\right)$ with

$$
\int_{z_{\not \supset 1} \in \mathbb{T}^{d-1}} g(s, x, z) \mathrm{d} z_{\not \supset 1}=0, \quad \text { for all }\left(s, x, z_{1}\right) .
$$

Define the function

$$
\rho\left(n, t, x, z_{1}\right)=g\left(t, x, z_{1}, n \omega_{2}\left(x+\left(c_{2}-c_{1}\right) t\right), \ldots, n \omega_{d}\left(x+\left(c_{d}-c_{1}\right) t\right)\right),
$$

where $\omega$ satisfies (8.1). Then, for each $R$ we have

$$
\sup \left\{\left|\int_{0}^{t} \rho\left(n, s, x, z_{1}\right) \mathrm{d} s\right|: t \in[0, R],|x| \leqslant R, z_{1} \in \mathbb{T}\right\} \rightarrow 0, \quad \text { for } n \rightarrow \infty .
$$

Proof. We proceed completely analogous to the above result. We first establish the result in the case that $g$ is $(d+1)$-times continuously differentiable with respect to $z_{\not \supset 1}$, such that for each $\left(t, x, z_{1}\right)$ a Fourier series expansion with the appropriate decay for the coefficients exists. This decay is uniform in $\left(t, x, z_{1}\right)$ for given $R$ as in the supremum above.

Now, the integrals over $s \in[0, t]$ can be modified by making the $s$ variable piecewise constant on intervals $I_{k}=[k \delta,(k+1) \delta]$. Then, integration over $s \in I_{k}$ can be performed on the Fourier series that shows, by using the small divisor estimate (8.1), that the integral over $I_{k}$ goes to zero for $n \rightarrow \infty$. It is important that all these considerations can be done uniformly in $\left(t, x, z_{1}\right)$.

Finally, for general not necessarily differentiable $g$ we approximate by sufficiently smooth functions and argue as in the proof of theorem (B.1).

\section{References}

1 R. Ash. Information theory (Wiley, 1965).

2 J. M. Ball. A version of the fundamental theorem for Young measures. In Proc. of the CNRS-NSF Workshop on Continuum Theory of Phase Transitions (ed. M. Rascle, D. Serre and M. Slemrod). Springer Lecture Notes in Physics, no. 344, pp. 207-221 (Springer, 1989).

3 J. Batt. Ein Existenzbeweis für die Vlasov-Gleichung der Stellardynamik bei gemittelter Dichte. Arch. Ration. Mech. Analysis 13 (1963), 296-308.

4 S. Demoulini. Young measure solutions for a nonlinear parabolic equation of forwardbackward type. SIAM J. Math. Analysis 27 (1996), 376-403.

5 R. J. DiPerna. Measure-valued solutions to conservation laws. Arch. Ration. Mech. Analysis 88 (1985), 223-270.

6 W. E and R. V. Kohn. The initial-value problem for measure-valued solutions of a canonical $2 \times 2$ system with linearly degenerate fields. Commun. Pure Appl. Math. 44 (1991), 9811000 .

7 I. Fonseca, D. Brandon and P. Swart. Dynamics and oscillatory microstructure in a model of displacive phase transformations. In Progress in partial differential equations: Metz surveys 3 (ed. M. Chipot, J. Saint Jean Paulin and I. Shafrir). Pitman Research Notes in Mathematics, vol. 314, pp. 130-144 (Pitman, 1994).

8 P. Gérard. Microlocal defect measures. Commun. Partial Diffl Eqns 16 (1991), 1761-1794.

9 R. T. Glassey. The cauchy problem in kinetic theory (SIAM, 1996). 
J. L. Joly, G. Métivier and J. Rauch. Resonant one-dimensional nonlinear geometric optics. J. Functional Analysis 114 (1993), 106-231.

11 J. L. Joly, G. Métivier and J. Rauch. Trilinear compensated compactness and nonlinear geometric optics. Ann. Math. 142 (1995), 121-169.

F. Murat. Compacité par compensation. Ann. Sci. Norm. Sup. Pisa 5 (1978), 489-507.

13 D. McLaughlin, G. Papanicolaou and L. Tartar. Weak limits of semilinear hyperbolic systems with oscillating data. In Macroscopic modelling of turbulent flows (ed. U. Frisch, J. B. Keller, G. Papanicolaou and O. Pironneau). Springer Lecture Notes in Physics no. 230, pp. 277-289 (Springer, 1985).

14 F. Otto. Dynamics of labyrinthine pattern formation in magnetic fluids: a mean-field theory. Arch. Ration. Mech. Analysis 141 (1998), 63-103.

15 S. T. Rachev. Probability metrics and the stability of stochastic models (Wiley, 1991).

16 C. L. Siegel and J. K. Moser. Lectures on celestial mechanics. Grundlehren, vol. 187 (Springer, 1971).

17 L. Tartar. Compensated compactness and applications to PDEs. In Nonlinear mechanics and analysis (ed. R. J. Knops). Pitman Reasearch Notes in Mathematics, vol. 4, pp. 136212 (Pitman, 1979).

18 L. Tartar. Solutions oscillantes des equations de Carleman. Seminaire Goulaouic-MeyerSchwartz, 1980-1981, Ecole Polytechnique (Palaiseau). Expsoe XII, 1981.

19 L. Tartar. The compensated compactness method applied to systems of conservation laws. In Systems of nonlinear PDE (ed. J. M. Ball), pp. 263-285 (D. Reidel, 1983).

20 L. Tartar. Etude des oscillations dans thes equations aux derivees partielles non lineaires. In Trends in applications of pure mathematics to mechanics (ed. M. Roseau and P. G. Ciarlet). Springer Lecture Notes in Physics, no. 195, pp. 384-412 (Springer, 1984).

21 L. Tartar. Oscillations and asymptotic behaviour of two semilinear hyperbolic systems. In Dynamics of infinite dimensional systems (ed. S. N. Chow and J. K. Hale), pp. 341-356 (Springer, 1987).

22 L. Tartar. $H$-measures, a new approach for studying homogenisation, oscillations and concentrations effects in partial differential equations. Proc. R. Soc. Edinb. A 115 (1990), 193-230.

23 L. Tartar. Oscillations for semilinear systems. Unpublished lecture notes, no. 91.3, 1991, 14 pp.

24 L. Tartar. Personal communication at Oberwolfach 1996.

25 F. Theil. Young-measure solutions for a microkinetically regularized wave equation. Draft. IfAM, Universität Hannover. September 1996.

26 F. Theil. Young measure solutions for a viscoelastically damped wave equation with nonmonotone stress-strain relation. Arch. Ration. Mech. Analysis 144 (1998), 47-78.

27 F. Theil and V. I. Levitas. A Hamiltonian model for martensitic phase transformations including microkinetic energy. Math. Mech. Solids. (Submitted.) 